First version: 13 April 2015; This version: 27 August 2016

\title{
REFLECTED BSDES WHEN THE OBSTACLE IS NOT RIGHT-CONTINUOUS AND OPTIMAL STOPPING
}

\author{
By Miryana Grigorova*, Peter Imkeller*, Elias Offen ${ }^{\dagger}$, Youssef Ouknine $^{\ddagger}$, \\ and Marie-Claire Quenez ${ }^{\S}$ \\ Humboldt University-Berlin ${ }^{*}$, University of Botswana ${ }^{\dagger}$, Université Cadi Ayyad ${ }^{\ddagger}$, and \\ Université Paris-Diderot $\S$
}

\begin{abstract}
In the first part of the paper, we study reflected backward stochastic differential equations (RBSDEs) with lower obstacle which is assumed to be right upper-semicontinuous but not necessarily right-continuous. We prove existence and uniqueness of the solutions to such RBSDEs in appropriate Banach spaces. The result is established by using some results from optimal stopping theory, some tools from the general theory of processes such as Mertens decomposition of optional strong supermartingales, as well as an appropriate generalization of Itô's formula due to Gal'chouk and Lenglart. In the second part of the paper, we provide some links between the RBSDE studied in the first part and an optimal stopping problem in which the risk of a financial position $\xi$ is assessed by an $f$-conditional expectation $\mathcal{E}^{f}(\cdot)$ (where $f$ is a Lipschitz driver). We characterize the "value function" of the problem in terms of the solution to our RBSDE. Under an additional assumption of left upper-semicontinuity along stopping times on $\xi$, we show the existence of an optimal stopping time. We also provide a generalization of Mertens decomposition to the case of strong $\mathcal{E}^{f}$-supermartingales.
\end{abstract}

1. Introduction. Backward stochastic differential equations (BSDEs) have been introduced by Bismut ([4]) in the case of a linear driver. The general theory of existence and uniqueness of solutions to BSDEs has been developed by Pardoux and Peng [29]. Through a result of Feynman-Kac-type, these authors have linked the theory of BSDEs to that of quasilinear parabolic partial differential equations (cf.[30]). BSDEs have found number of applications in finance, among which pricing and hedging of European options and recursive utilities (cf., for instance, [13], [14]). Also, a useful family of operators, the family of so-called $f$-conditional expectations, has been defined through the notion of BSDEs and used in the literature on dynamic risk measures (cf., for instance, [2], [32], [33], [35], [3] among others).

MSC 2010 subject classifications: Primary 60G40, 93E20, 60H30; secondary 60G07, 47N10

Keywords and phrases: backward stochastic differential equation, reflected backward stochastic differential equation, optimal stopping, $f$-expectation, strong optional supermartingale, Mertens decomposition, dynamic risk measure, strong $\mathcal{E}^{f}$-supermartingale 
We recall that the $f$-conditional expectation at time $t \in[0, T]$ (where $T>0$ is a fixed final horizon) is the operator which maps a given square-integrable terminal condition $\xi_{T}$ to the position at time $t$ of (the first component of) the solution to the BSDE with parameters $\left(f, \xi_{T}\right)$. The operator is denoted $\mathcal{E}_{t, T}^{f}(\cdot)$.

Reflected backward stochastic differential equations (RBSDEs) can be seen as a variant of BSDEs in which the (first component of the) solution is constrained to remain greater than or equal to a given process called the obstacle. Compared to the case of (non-reflected) BSDEs, there is an additional nondecreasing predictable process which keeps the (first component of the) solution above the obstacle.

RBSDEs have been introduced by El Karoui et al. [12] in the case of a Brownian filtration and a continuous obstacle. In [14], El Karoui and Quenez also study their links with the (non linear) pricing of American options. There have been several extensions of these works to the case of a discontinuous obstacle and/or a larger stochastic basis than the Brownian one (cf. [18], [6], [19], [15], [20], [34]). In all these extensions an assumption of right-continuity on the obstacle is made.

In the first part of the present paper we consider a further extension of the theory of RBSDEs to the case where the obstacle is not necessarily right-continuous. Compared to the right-continuous case, the additional nondecreasing process, which "pushes" the (first component of the) solution to stay above the obstacle, is no longer right-continuous. To prove our results we use some tools from the optimal stopping theory (cf. [27], [11], [21], [22]), some tools from the general theory of processes (cf. [9]) such as Mertens decomposition of strong optional (but not necessarily right-continuous) supermartingales (generalizing DoobMeyer decomposition), a result from the potential theory (cf. [9]), and a generalization of Itô's formula to the case of strong optional semimartingales in the vocabulary of [16] (but not necessarily right-continuous) due to Gal'chouk and Lenglart (cf. [26]).

In the second part of the paper, we make some links between the RBSDEs studied in the first part and optimal stopping with $f$-conditional expectations. More precisely, we are interested in the following optimization problem: we are given a process $\xi$ modelling a dynamic financial position. The risk of $\xi$ is assessed by a dynamic risk measure which (up to a minus sign) is given by an $f$-conditional expectation. The process $\xi$ is assumed to be right upper-semicontinuous, but not necessarily right-continuous. We aim at stopping the process $\xi$ in such a way that the risk be minimal. We characterize the value of the problem in terms of the unique solution to the RBSDE associated with obstacle $\xi$ and driver $f$ studied in the first part. We show the existence of an optimal stopping time for the problem under an additional assumption of left upper-semicontinuity along stopping times on $\xi$, and the existence of an $\varepsilon$-optimal stopping time in the more general case where this assumption is not made. We provide an optimality criterion characterizing the optimal stopping times for 
the problem in terms of properties of the "value process". We thus extend some results of [34] to the case where the optimized process $\xi$ is not cadlag. We also establish a comparison principle for the RBSDEs studied in the first part of our paper, as well as a generalization of Mertens decomposition to the case of $\mathcal{E}^{f}$-strong supermartingales.

The remainder of the paper is organized as follows:

In Section 2 we give some preliminary definitions and properties. In Section 3 we define our RBSDE and we prove existence and uniqueness of the solution. Section 4 is dedicated to our optimal stopping problem with $f$-conditional expectations. In Subsection 4.1 we formulate and motivate the problem. In Subsection 4.2 we characterize the value function of the problem in terms of the solution of the RBSDE studied in Section 3; we also give an optimality criterion and address the question of existence of $\varepsilon$-optimal and optimal stopping times. In Section 5 we derive some useful additional results: comparison principle for our RBSDEs (Subsection 5.2) and "generalized" Mertens decomposition for $\mathcal{E}^{f}$-strong supermartingales (Subsection 5.1). In Section 6 we briefly present some further extensions of our work. In the Appendix we recall some useful results ("classical" Mertens decomposition, a result from potential theory, Gal'chouk-Lenglart's change of variables formula), we give the proofs of three results (Prop. 2.1, Prop. A.6, and Prop. A.5) used in the main part of the paper, and we also give some examples.

2. Preliminaries. Let $T>0$ be a fixed positive real number. Let $(E, \mathscr{E})$ be a measurable space equipped with a $\sigma$-finite positive measure $\nu$. Let $(\Omega, \mathcal{F}, P)$ be a probability space equipped with a one-dimensional Brownian motion $W$ and with an independent Poisson random measure $N(d t, d e)$ with compensator $d t \otimes \nu(d e)$. We denote by $\tilde{N}(d t, d e)$ the compensated process, i.e. $\tilde{N}(d t, d e):=N(d t, d e)-d t \otimes \nu(d e)$. Let $\mathbb{F}=\left\{\mathcal{F}_{t}: t \in[0, T]\right\}$ be the (complete) natural filtration associated with $W$ and $N$. For $t \in[0, T]$, we denote by $\mathcal{T}_{t, T}$ the set of stopping times $\tau$ such that $P(t \leq \tau \leq T)=1$. More generally, for a given stopping time $\nu \in \mathcal{T}_{0, T}$, we denote by $\mathcal{T}_{\nu, T}$ the set of stopping times $\tau$ such that $P(\nu \leq \tau \leq T)=1$.

We use the following notation:

- $\mathcal{P}$ is the predictable $\sigma$-algebra on $\Omega \times[0, T]$.

- Prog is the progressive $\sigma$-algebra on $\Omega \times[0, T]$.

- $\mathcal{B}(\mathbf{R})\left(\right.$ resp. $\mathcal{B}\left(\mathbf{R}^{2}\right)$ ) is the Borel $\sigma$-algebra on $\mathbf{R}$ (resp. $\left.\mathbf{R}^{2}\right)$.

- $L^{2}\left(\mathcal{F}_{T}\right)$ is the set of random variables which are $\mathcal{F}_{T}$-measurable and square-integrable.

- $L_{\nu}^{2}$ is the set of $(\mathscr{E}, \mathcal{B}(\mathbf{R}))$-measurable functions $\ell: E \rightarrow \mathbf{R}$ such that $\|\ell\|_{\nu}^{2}:=$ $\int_{E}|\ell(e)|^{2} \nu(d e)<\infty$. For $\ell \in \mathcal{L}_{\nu}^{2}, \mathcal{K} \in \mathcal{L}_{\nu}^{2}$, we define $\langle\ell, \kappa\rangle_{\nu}:=\int_{E} \ell(e) \kappa(e) \nu(d e)$.

- $\mathcal{B}\left(L_{\nu}^{2}\right)$ is the Borel $\sigma$-algebra on $L_{\nu}^{2}$.

- $\mathbb{I H}^{2}$ is the set of $\mathbf{R}$-valued predictable processes $\phi$ with $\|\phi\|_{H^{2}}^{2}:=E\left[\int_{0}^{T}\left|\phi_{t}\right|^{2} d t\right]<\infty$. 
- $\mathbb{H}_{\nu}^{2}$ is the set of $\mathbf{R}$-valued processes $l:(\omega, t, e) \in(\Omega \times[0, T] \times E) \mapsto l_{t}(\omega, e)$ which are predictable, that is $(\mathcal{P} \otimes \mathscr{E}, \mathcal{B}(\mathbf{R}))$-measurable, and such that $\|l\|_{\mathbb{H}_{\nu}^{2}}^{2}:=$ $E\left[\int_{0}^{T}\left\|l_{t}\right\|_{\nu}^{2} d t\right]<\infty$.

We introduce the vector space $\mathcal{S}^{2}$ defined as the space of $\mathbf{R}$-valued optional (not necessarily cadlag) processes $\phi$ such that $\|\phi\|_{\mathcal{S}^{2}}^{2}:=E\left[\operatorname{ess}_{\sup } \operatorname{si\mathcal {T}}_{0, T}\left|\phi_{\tau}\right|^{2}\right]<\infty .{ }^{1}$

Proposition 2.1 The map $\|\cdot\|_{\mathcal{S}^{2}}$ is a norm on $\mathcal{S}^{2}$. In particular, if $\phi \in \mathcal{S}^{2}$ is such that $\|\phi\|_{\mathcal{S}^{2}}^{2}=0$, then $\phi$ is indistinguishable from the null process, that is $\phi_{t}=0,0 \leq t \leq T$ a.s. Moreover, the space $\mathcal{S}^{2}$ endowed with the norm $\|\cdot \mid\|_{\mathcal{S}^{2}}$ is a Banach space.

The proof is given in the Appendix.

We will also use the following notation:

Let $\beta>0$. For $\phi \in \mathbb{H}^{2},\|\phi\|_{\beta}^{2}:=E\left[\int_{0}^{T} \mathrm{e}^{\beta s} \phi_{s}^{2} d s\right]$. We note that on the space $\mathbb{H}^{2}$ the norms $\|\cdot\|_{\beta}$ and $\|\cdot\|_{\mathbb{H}^{2}}$ are equivalent. For $l \in \mathbb{H}_{\nu}^{2},\|l\|_{\nu, \beta}^{2}:=E\left[\int_{0}^{T} \mathrm{e}^{\beta s}\left\|l_{s}\right\|_{\nu}^{2} d s\right]$. On the space $\mathbb{H}_{\nu}^{2}$ the norms $\|\cdot\|_{\nu, \beta}$ and $\|\cdot\|_{\mathbb{H}_{\nu}^{2}}$ are equivalent. For $\phi \in \mathcal{S}^{2}$, we define $\|\phi\|_{\beta}^{2}:=$ $E\left[\operatorname{ess} \sup _{\tau \in \mathcal{T}_{0, T}} \mathrm{e}^{\beta \tau} \phi_{\tau}^{2}\right]$. We note that $\|\cdot\| \|_{\beta}$ is a norm on $\mathcal{S}^{2}$ equivalent to the norm $\|\cdot \cdot\|_{\mathcal{S}^{2}}$.

Remark 2.1 By a slight abuse of notation, we shall also write $\|\phi\|_{H^{2}}^{2}$ (resp. $\|\phi\|_{\beta}^{2}$ ) for $E\left[\int_{0}^{T}\left|\phi_{t}\right|^{2} d t\right]$ (resp. $E\left[\int_{0}^{T} \mathrm{e}^{\beta t}\left|\phi_{t}\right|^{2} d t\right]$ ) in the case of a progressively measurable real-valued process $\phi$ (cf., e.g., Remark 2.1 in [6] for the same notation).

Definition 2.1 (Driver, Lipschitz driver) A function $f$ is said to be a driver if

- $f: \Omega \times[0, T] \times \mathbf{R}^{2} \times L_{\nu}^{2} \rightarrow \mathbf{R}$ $(\omega, t, y, z, \mathcal{K}) \mapsto f(\omega, t, y, z, \mathcal{K})$ is $\operatorname{Prog} \otimes \mathcal{B}\left(\mathbf{R}^{2}\right) \otimes \mathcal{B}\left(L_{\nu}^{2}\right)-$ measurable, - $E\left[\int_{0}^{T} f(t, 0,0,0)^{2} d t\right]<+\infty$.

A driver $f$ is called a Lipschitz driver if moreover there exists a constant $K \geq 0$ such that $d P \otimes d t$-a.e., for each $\left(y_{1}, z_{1}, k_{1}\right) \in \mathbf{R}^{2} \times L_{\nu}^{2},\left(y_{2}, z_{2}, k_{2}\right) \in \mathbf{R}^{2} \times L_{\nu}^{2}$,

$$
\left|f\left(\omega, t, y_{1}, z_{1}, k_{1}\right)-f\left(\omega, t, y_{2}, z_{2}, k_{2}\right)\right| \leq K\left(\left|y_{1}-y_{2}\right|+\left|z_{1}-z_{2}\right|+\left\|k_{1}-k_{2}\right\|_{\nu}\right) .
$$

A Lipschitz driver $f$ is called predictable if moreover $f$ is $\mathcal{P} \otimes \mathcal{B}\left(\mathbf{R}^{2}\right) \otimes \mathcal{B}\left(L_{\nu}^{2}\right)-$ measurable.

For real-valued random variables $X$ and $X_{n}, n \in \mathbb{N}$, the notation " $X_{n} \uparrow X^{\text {" will stand }}$ for "the sequence $\left(X_{n}\right)$ is nondecreasing and converges to $X$ a.s.".

For a ladlag process $\phi$, we denote by $\phi_{t+}$ and $\phi_{t-}$ the right-hand and left-hand limit of $\phi$ at $t$. We denote by $\Delta_{+} \phi_{t}:=\phi_{t_{+}}-\phi_{t}$ the size of the right jump of $\phi$ at $t$, and by $\Delta \phi_{t}:=\phi_{t}-\phi_{t-}$ the size of the left jump of $\phi$ at $t$.

\footnotetext{
${ }^{1}$ Note that when $\phi$ is right-continuous, $\|\mid \phi\|_{\mathcal{S}^{2}}^{2}=E\left[\sup _{t \in[0, T]}\left|\phi_{t}\right|^{2}\right]$ (cf. Remark A.14).
} 
Definition 2.2 Let $\tau \in \mathcal{T}_{0, T}$. An optional process $\left(\phi_{t}\right)$ is said to be right upper-semicontinuous (r.u.s.c.) (resp. left upper-semicontinuous (l.u.s.c.)) along stopping times at the stopping time $\tau$ if for all nonincreasing (resp. nondecreasing) sequence of stopping times $\left(\tau_{n}\right)$ such that $\tau^{n} \downarrow \tau$ (resp. $\tau^{n} \uparrow \tau$ ) a.s., $\phi_{\tau} \geq \lim \sup _{n \rightarrow \infty} \phi_{\tau_{n}}$ a.s.. The process $\left(\phi_{t}\right)$ is said to be r.u.s.c. (resp. l.u.s.c.) along stopping times if it is r.u.s.c. (resp. l.u.s.c.) along stopping times at each $\tau \in \mathcal{T}_{0, T}$. The right-(resp. left-) continuity property of an optional process $\left(\phi_{t}\right)$ along stopping times at a stopping time $\tau$ is defined similarly.

Remark 2.2 Note that if $\left(\phi_{t}\right)$ is an optional process and $\tau$ is a totally inaccessible stopping time, then $\left(\phi_{t}\right)$ is left-continuous along stopping times at $\tau$.

If the process $\left(\phi_{t}\right)$ has left limits, $\left(\phi_{t}\right)$ is l.u.s.c. (resp. left-continuous) along stopping times if and only if for each predictable stopping time $\tau \in \mathcal{T}_{0, T}, \phi_{\tau-} \leq \phi_{\tau}$ (resp. $\left.\phi_{\tau-}=\phi_{\tau}\right)$ a.s.

Note, moreover, that if an optional process $\left(\phi_{t}\right)$ is right upper-semicontinuous (r.u.s.c.), then it is r.u.s.c. along stopping times. The converse also holds true; it is a difficult result of the general theory of processes proved in [7, Prop. 2, page 300].

3. Reflected BSDE whose obstacle is not cadlag. Let $T>0$ be a fixed terminal time. Let $f$ be a driver. Let $\xi=\left(\xi_{t}\right)_{t \in[0, T]}$ be a left-limited process in $\mathcal{S}^{2}$. We suppose moreover that the process $\xi$ is r.u.s.c. A process $\xi$ satisfying the previous properties will be called a barrier, or an obstacle.

Remark 3.3 Let us note that in the following definitions and results we can relax the assumption of existence of left limits for the obstacle $\xi$. All the results still hold true provided we replace the process $\left(\xi_{t-}\right)_{t \in] 0, T]}$ by the process $\left(\underline{\xi}_{t}\right)_{t \in] 0, T]}$ defined by $\underline{\xi}_{t}:=\lim \sup _{s \uparrow t, s<t} \xi_{s}$, for all $t \in] 0, T]$. We recall that $\underline{\xi}$ is a predictable process (cf. [8, Thm. 90, page 225]). We call the process $\underline{\xi}$ the left upper-semicontinuous envelope of $\xi$.

Definition 3.1 A process $(Y, Z, k, A, C)$ is said to be a solution to the reflected BSDE with 
parameters $(f, \xi)$, where $f$ is a driver and $\xi$ is an obstacle, if

$(Y, Z, k, A, C) \in \mathcal{S}^{2} \times \mathbb{H}^{2} \times \mathbb{H}_{\nu}^{2} \times \mathcal{S}^{2} \times \mathcal{S}^{2}$ and a.s. for all $t \in[0, T]$

$Y_{t}=\xi_{T}+\int_{t}^{T} f\left(s, Y_{s}, Z_{s}, k_{s}\right) d s-\int_{t}^{T} Z_{s} d W_{s}-\int_{t}^{T} \int_{E} k_{s}(e) \tilde{N}(d s, d e)+A_{T}-A_{t}+C_{T-}-C_{t-}$,

$Y_{t} \geq \xi_{t}$ for all $t \in[0, T]$ a.s.

$A$ is a nondecreasing right-continuous predictable process with $A_{0}=0$ and such that

$\int_{0}^{T} \mathbf{1}_{\left\{Y_{t}>\xi_{t}\right\}} d A_{t}^{c}=0$ a.s. and $\left(Y_{\tau-}-\xi_{\tau-}\right)\left(A_{\tau}^{d}-A_{\tau-}^{d}\right)=0$ a.s. for all predictable $\tau \in \mathcal{T}_{0, T}$, $C$ is a nondecreasing right-continuous adapted purely discontinuous process with $C_{0-}=0$

and such that $\left(Y_{\tau}-\xi_{\tau}\right)\left(C_{\tau}-C_{\tau-}\right)=0$ a.s. for all $\tau \in \mathcal{T}_{0, T}$.

Here $A^{c}$ denotes the continuous part of the process $A$ and $A^{d}$ its discontinuous part.

Equations (3.3) and (3.4) are referred to as minimality conditions or Skorokhod conditions. We note that, by a classical result of the general theory of processes ([8, Theorem IV.84]), a process $(Y, Z, k, A, C) \in \mathcal{S}^{2} \times \mathbb{H}^{2} \times \mathbb{H}_{\nu}^{2} \times \mathcal{S}^{2} \times \mathcal{S}^{2}$ satisfies equation (3.1) in the above definition if and only if $Y_{\tau}=\xi_{T}+\int_{\tau}^{T} f\left(t, Y_{t}, Z_{t}, k_{t}\right) d t-\int_{\tau}^{T} Z_{t} d W_{t}-\int_{\tau}^{T} \int_{E} k_{t}(e) \tilde{N}(d t, d e)+$ $A_{T}-A_{\tau}+C_{T-}-C_{\tau-}$, where the equality holds a.s. for all $\tau \in \mathcal{T}_{0, T}$. Let us also emphasize that if $(Y, Z, k, A, C)$ satisfies the above definition, then the process $Y$ has left and right limits.

Remark 3.4 If $(Y, Z, k, A, C)$ is a solution to the RBSDE defined above, then $\Delta C_{t}(\omega)=$ $Y_{t}(\omega)-Y_{t+}(\omega)$ for all $(\omega, t) \in \Omega \times[0, T)$ outside an evanescent set. This observation is a consequence of equation (3.1). It follows that $Y_{t} \geq Y_{t+}$, for all $t \in[0, T)$, which implies that $Y$ is necessarily r.u.s.c.

Moreover, since in our framework the filtration is quasi-left-continuous, martingales have only totally inaccessible jumps. Hence, if $\tau$ is a predictable stopping time, we have $Y_{\tau}-Y_{\tau-}=$ $-\left(A_{\tau}-A_{\tau-}\right)$ a.s. From this, together with Remark 2.2, it follows that the process $Y$ is leftcontinuous along stopping times at a stopping time $\tau$ if and only if $\Delta A_{\tau}=0$ a.s.

We also note that equality (3.1) still holds with $f\left(t, Y_{t}, Z_{t}, k_{t}\right)$ replaced by $f\left(t, Y_{t-}, Z_{t}, k_{t}\right)$. Furthermore, the process $\left(Y_{t}+\int_{0}^{t} f\left(s, Y_{s}, Z_{s}, k_{s}\right) d s\right)_{t \in[0, T]}$ is a strong supermartingale (cf. Definition A.1).

Remark 3.5 (the particular case of a right-continuous obstacle) In the particular 
case of a right-continuous obstacle $\xi$, we have that $Y$ is right-continuous. Indeed, observe that $Y_{t} \geq Y_{t+} \geq \xi_{t+}=\xi_{t}$ (due to the right upper semicontinuity of $Y$ and to inequality (3.2)). Hence, if $t$ is such that $Y_{t}=\xi_{t}$, then $Y_{t}=Y_{t+}=\xi_{t}$. If $t$ is such that $Y_{t}>\xi_{t}$, then $Y_{t}-Y_{t+}=C_{t}-C_{t-}=0$ (due to Remark 3.4 and to (3.4)). Thus, in both cases, $Y_{t}=Y_{t+}$; so, $Y$ is right-continuous.

Moreover, the right-continuity of $Y$ combined with Remark 3.4 give $C_{t}=C_{t-}$, for all $t$. As $C$ is right-continuous, purely discontinuous and such that $C_{0-}=0$, we deduce $C \equiv 0$. Thus, we recover the usual formulation of RBSDE with right-continuous obstacle.

A simple introductory example where a solution to our RBSDE (from Definition 3.1) can be explicitly computed is presented in the Appendix (cf. Example A.1).

Let us now investigate the question of existence and uniqueness of the solution to the RBSDE defined above in the case where the driver $f$ does not depend on $y, z$, and $k$. To this purpose, we first state a lemma which will be used in the sequel.

Lemma 3.2 (A priori estimates) Let $\left(Y^{1}, Z^{1}, k^{1}, A^{1}, C^{1}\right) \in \mathcal{S}^{2} \times \mathbb{H}^{2} \times \mathbb{H}_{\nu}^{2} \times \mathcal{S}^{2} \times \mathcal{S}^{2}$ (resp. $\left(Y^{2}, Z^{2}, k^{2}, A^{2}, C^{2}\right) \in \mathcal{S}^{2} \times \mathbb{H}^{2} \times \mathbb{H}_{\nu}^{2} \times \mathcal{S}^{2} \times \mathcal{S}^{2}$ ) be a solution to the RBSDE associated with driver $f^{1}(\omega, t)$ (resp. $\left.f^{2}(\omega, t)\right)$ and with obstacle $\xi$. There exists $c>0$ such that for all $\varepsilon>0$, for all $\beta \geq \frac{1}{\varepsilon^{2}}$ we have

$$
\begin{aligned}
\left\|k^{1}-k^{2}\right\|_{\nu, \beta}^{2} \leq \varepsilon^{2}\left\|f^{1}-f^{2}\right\|_{\beta}^{2} ; & \left\|Z^{1}-Z^{2}\right\|_{\beta}^{2} \leq \varepsilon^{2}\left\|f^{1}-f^{2}\right\|_{\beta}^{2} ; \\
\left\|Y^{1}-Y^{2}\right\|_{\beta}^{2} & \leq 4 \varepsilon^{2}\left(1+6 c^{2}\right)\left\|f^{1}-f^{2}\right\|_{\beta}^{2} .
\end{aligned}
$$

Proof of Lemma 3.2: Let $\beta>0$ and $\varepsilon>0$ be such that $\beta \geq \frac{1}{\varepsilon^{2}}$. We set $\tilde{Y}:=Y^{1}-Y^{2}$, $\tilde{Z}:=Z^{1}-Z^{2}, \tilde{A}:=A^{1}-A^{2}, \tilde{C}:=C^{1}-C^{2}, \tilde{k}:=k^{1}-k^{2}$, and $\tilde{f}(\omega, t):=f^{1}(\omega, t)-f^{2}(\omega, t)$. We note that $\tilde{Y}_{T}=\xi_{T}-\xi_{T}=0$; moreover,

$\tilde{Y}_{\tau}=\int_{\tau}^{T} \tilde{f}(t) d t-\int_{\tau}^{T} \tilde{Z}_{t} d W_{t}-\int_{\tau}^{T} \int_{E} \tilde{k}_{t}(e) \tilde{N}(d t, d e)+\tilde{A}_{T}-\tilde{A}_{\tau}+\tilde{C}_{T-}-\tilde{C}_{\tau-}$ a.s. for all $\tau \in \mathcal{T}_{0, T}$.

Thus we see that $\tilde{Y}$ is an optional strong semimartingale in the vocabulary of [16] (cf. Theorem A.3 for the definition) with decomposition $\tilde{Y}=\tilde{Y}_{0}+M+A+B$, where $M_{t}:=$ $\int_{0}^{t} \tilde{Z}_{s} d W_{s}+\int_{0}^{t} \int_{E} \tilde{k}_{s}(e) \tilde{N}(d s, d e), A_{t}:=-\int_{0}^{t} \tilde{f}(s) d s-\tilde{A}_{t}$ and $B_{t}:=-\tilde{C}_{t-}$ (the notation is that of Theorem A.3 and Corollary A.2 from the Appendix). Applying Gal'chouk-Lenglart's 
formula (more precisely Corollary A.2) to $\mathrm{e}^{\beta t} \tilde{Y}_{t}^{2}$ gives: almost surely, for all $t \in[0, T]$,

$$
\begin{aligned}
\mathrm{e}^{\beta T} \tilde{Y}_{T}^{2} & =\mathrm{e}^{\beta t} \tilde{Y}_{t}^{2}+\int_{] t, T]} \beta \mathrm{e}^{\beta s}\left(\tilde{Y}_{s}\right)^{2} d s-2 \int_{] t, T]} \mathrm{e}^{\beta s} \tilde{Y}_{s-} \tilde{f}(s) d s-2 \int_{] t, T]} \mathrm{e}^{\beta s} \tilde{Y}_{s-} d \tilde{A}_{s} \\
& +2 \int_{] t, T]} \mathrm{e}^{\beta s} \tilde{Y}_{s-} \tilde{Z}_{s} d W_{s}+2 \int_{] t, T]} \mathrm{e}^{\beta s} \int_{E} \tilde{Y}_{s-} \tilde{k}_{s}(e) \tilde{N}(d s, d e)+\int_{] t, T]} \mathrm{e}^{\beta s} \tilde{Z}_{s}^{2} d s \\
& +\sum_{t<s \leq T} \mathrm{e}^{\beta s}\left(\tilde{Y}_{s}-\tilde{Y}_{s-}\right)^{2}-\int_{[t, T[} 2 \mathrm{e}^{\beta s} \tilde{Y}_{s} d(\tilde{C})_{s+}+\sum_{t \leq s<T} \mathrm{e}^{\beta s}\left(\tilde{Y}_{s+}-\tilde{Y}_{s}\right)^{2} .
\end{aligned}
$$

Thus, we get (recall that $\tilde{Y}_{T}=0$ ): almost surely, for all $t \in[0, T]$,

$$
\begin{aligned}
\mathrm{e}^{\beta t} \tilde{Y}_{t}^{2}+\int_{] t, T]} \mathrm{e}^{\beta s} \tilde{Z}_{s}^{2} d s & =-\int_{] t, T]} \beta \mathrm{e}^{\beta s}\left(\tilde{Y}_{s}\right)^{2} d s+2 \int_{] t, T]} \mathrm{e}^{\beta s} \tilde{Y}_{s} \tilde{f}(s) d s+2 \int_{] t, T]} \mathrm{e}^{\beta s} \tilde{Y}_{s-} d \tilde{A}_{s} \\
& -2 \int_{] t, T]} \mathrm{e}^{\beta s} \tilde{Y}_{s-} \tilde{Z}_{s} d W_{s}-2 \int_{] t, T]} \mathrm{e}^{\beta s} \int_{E} \tilde{Y}_{s-} \tilde{k}_{s}(e) \tilde{N}(d s, d e) \\
& -\sum_{t<s \leq T} \mathrm{e}^{\beta s}\left(\tilde{Y}_{s}-\tilde{Y}_{s-}\right)^{2}+2 \int_{[t, T[} \mathrm{e}^{\beta s} \tilde{Y}_{s} d \tilde{C}_{s}-\sum_{t \leq s<T} \mathrm{e}^{\beta s}\left(\tilde{Y}_{s+}-\tilde{Y}_{s}\right)^{2} .
\end{aligned}
$$

We give hereafter an upper bound for some of the terms appearing on the right-hand side (r.h.s. for short) of the above equality.

Let us first consider the sum of the first and the second term on the r.h.s. of equality (3.6). By applying the inequality $2 a b \leq\left(\frac{a}{\varepsilon}\right)^{2}+\varepsilon^{2} b^{2}$, valid for all $(a, b) \in \mathbf{R}^{2}$, we get: a.s. for all $t \in[0, T]$,

$$
\begin{aligned}
-\int_{] t, T]} \beta \mathrm{e}^{\beta s}\left(\tilde{Y}_{s}\right)^{2} d s+2 \int_{] t, T]} \mathrm{e}^{\beta s} \tilde{Y}_{s} \tilde{f}(s) d s \leq & -\int_{] t, T]} \beta \mathrm{e}^{\beta s}\left(\tilde{Y}_{s}\right)^{2} d s+\frac{1}{\varepsilon^{2}} \int_{] t, T]} \mathrm{e}^{\beta s} \tilde{Y}_{s}^{2} d s \\
& +\varepsilon^{2} \int_{] t, T]} \mathrm{e}^{\beta s} \tilde{f}^{2}(s) d s \\
= & \left(\frac{1}{\varepsilon^{2}}-\beta\right) \int_{] t, T]} \mathrm{e}^{\beta s}\left(\tilde{Y}_{s}\right)^{2} d s+\varepsilon^{2} \int_{] t, T]} \mathrm{e}^{\beta s} \tilde{f}^{2}(s) d s .
\end{aligned}
$$

As $\beta \geq \frac{1}{\varepsilon^{2}}$, we have $\left(\frac{1}{\varepsilon^{2}}-\beta\right) \int_{\mid t, T]} \mathrm{e}^{\beta s}\left(\tilde{Y}_{s}\right)^{2} d s \leq 0$, for all $t \in[0, T]$ a.s.

For the third term on the r.h.s. of (3.6) it can be shown that, a.s. for all $t \in[0, T]$, $2 \int_{] t, T]} \mathrm{e}^{\beta s} \tilde{Y}_{s-} d \tilde{A}_{s} \leq 0$. The proof uses property (3.3) of the definition of the RBSDE and the property $Y^{i} \geq \xi$, for $i=1,2$; the details are similar to those in the case of a cadlag obstacle and are left to the reader (cf., for instance, [34, proof of Prop. A.1]).

For the the last but one term on the r.h.s. of (3.6) we show that, a.s. for all $t \in[0, T]$, $2 \int_{[t, T[} \mathrm{e}^{\beta s} \tilde{Y}_{s} d \tilde{C}_{s} \leq 0$. Indeed, a.s. for all $t \in[0, T], \int_{[t, T[} \mathrm{e}^{\beta s} \tilde{Y}_{s} d \tilde{C}_{s}=\sum_{t \leq s<T} \mathrm{e}^{\beta s} \tilde{Y}_{s} \Delta \tilde{C}_{s}$. Now, a.s. for all $s \in[0, T], \tilde{Y}_{s} \Delta \tilde{C}_{s}=\left(Y_{s}^{1}-Y_{s}^{2}\right) \Delta C_{s}^{1}-\left(Y_{s}^{1}-Y_{s}^{2}\right) \Delta C_{s}^{2}$. We use property 
(3.4), the non-decreasingness of (almost all trajectories of) $C^{1}$, and the fact that $Y^{2} \geq \xi$ to obtain: a.s. for all $s \in[0, T]$,

$$
\left(Y_{s}^{1}-Y_{s}^{2}\right) \Delta C_{s}^{1}=\left(Y_{s}^{1}-\xi_{s}\right) \Delta C_{s}^{1}-\left(Y_{s}^{2}-\xi_{s}\right) \Delta C_{s}^{1}=-\left(Y_{s}^{2}-\xi_{s}\right) \Delta C_{s}^{1} \leq 0 .
$$

Similarly, we obtain: a.s. for all $s \in[0, T],\left(Y_{s}^{1}-Y_{s}^{2}\right) \Delta C_{s}^{2}=\left(Y_{s}^{1}-\xi_{s}\right) \Delta C_{s}^{2} \geq 0$. We conclude that, a.s. for all $t \in[0, T], 2 \int_{[t, T]} \mathrm{e}^{\beta s} \tilde{Y}_{s} d \tilde{C}_{s} \leq 0$. The above observations, together with equation (3.6), lead to the following inequality: a.s., for all $t \in[0, T]$,

$$
\begin{aligned}
\mathrm{e}^{\beta t} \tilde{Y}_{t}^{2}+\int_{] t, T]} \mathrm{e}^{\beta s} \tilde{Z}_{s}^{2} d s & \leq \varepsilon^{2} \int_{] t, T]} \mathrm{e}^{\beta s} \tilde{f}^{2}(s) d s-2 \int_{] t, T]} \mathrm{e}^{\beta s} \tilde{Y}_{s-} \tilde{Z}_{s} d W_{s} \\
& -2 \int_{] t, T]} \mathrm{e}^{\beta s} \int_{E} \tilde{Y}_{s-} \tilde{k}_{s}(e) \tilde{N}(d s, d e)-\sum_{t<s \leq T} \mathrm{e}^{\beta s}\left(\tilde{Y}_{s}-\tilde{Y}_{s-}\right)^{2} .
\end{aligned}
$$

From the above inequality we derive first an estimate for $\|\tilde{Z}\|_{\beta}^{2}$ and $\|\tilde{k}\|_{\nu, \beta}^{2}$, and then an estimate for $\|\tilde{Y}\|_{\beta}^{2}$.

Estimate for $\|\tilde{Z}\|_{\beta}^{2}$ and $\|\tilde{k}\|_{\nu, \beta}^{2}$. Note first that we have:

$$
\begin{aligned}
\int_{] t, T]} \mathrm{e}^{\beta s}\left\|\tilde{k}_{s}\right\|_{\nu}^{2} d s-\sum_{t<s \leq T} \mathrm{e}^{\beta s}\left(\tilde{Y}_{s}-\tilde{Y}_{s-}\right)^{2}= & \int_{] t, T]} \mathrm{e}^{\beta s}\left\|\tilde{k}_{s}\right\|_{\nu}^{2} d s-\int_{] t, T]} \mathrm{e}^{\beta s} \int_{E} \tilde{k}_{s}^{2}(e) N(d s, d e) \\
& -\sum_{t<s \leq T} \mathrm{e}^{\beta s}\left(\Delta \tilde{A}_{s}\right)^{2} \\
= & -\sum_{t \leq s<T} \mathrm{e}^{\beta s} \Delta \tilde{A}_{s}^{2}-\int_{] t, T]} \mathrm{e}^{\beta s} \int_{E} \tilde{k}_{s}^{2}(e) \tilde{N}(d s, d e),
\end{aligned}
$$

where, in order to obtain the first equality, we have used the fact that the processes $A$. and $N(\cdot, d e)$ "do not have jumps in common" (recall that the process $A$ jumps only at predictable stopping times, while the process $N(\cdot, d e)$ does not jump at predictable stopping times). By adding the term $\int_{] t, T]} \mathrm{e}^{\beta s}\left\|\tilde{k}_{s}\right\|_{\nu}^{2} d s$ on both sides of inequality (3.7) and by using the above computation, we derive that almost surely, for all $t \in[0, T]$,

$$
\begin{aligned}
\mathrm{e}^{\beta t} \tilde{Y}_{t}^{2}+\int_{] t, T]} \mathrm{e}^{\beta s} \tilde{Z}_{s}^{2} d s+\int_{] t, T]} \mathrm{e}^{\beta s}\left\|\tilde{k}_{s}\right\|_{\nu}^{2} d s & \leq \varepsilon^{2} \int_{] t, T]} \mathrm{e}^{\beta s} \tilde{f}^{2}(s) d s-2 \int_{] t, T]} \mathrm{e}^{\beta s} \tilde{Y}_{s-} \tilde{Z}_{s} d W_{s} \\
& -\int_{] t, T]} \mathrm{e}^{\beta s} \int_{E}\left(2 \tilde{Y}_{s-} \tilde{k}_{s}(e)+\tilde{k}_{s}^{2}(e)\right) \tilde{N}(d s, d e) .
\end{aligned}
$$

Let us show that the stochastic integral "with respect to $d W_{s}$ " has zero expectation. Note first that

$$
\sup _{t \in] 0, T]} \tilde{Y}_{t-}^{2}=\sup _{t \in \mathbb{Q} \cap] 0, T]} \tilde{Y}_{t-}^{2} \leq \operatorname{esssup}_{\tau \in \mathcal{T}_{0, T}} \tilde{Y}_{\tau}^{2} \quad \text { a.s }
$$


where we have used the left-continuity of the process $\left(\tilde{Y}_{s-}\right)$ to obtain the equality. From this property together with Cauchy-Schwarz inequality, we get $E\left[\sqrt{\int_{0}^{T} \mathrm{e}^{2 \beta s} \tilde{Y}_{s-}^{2} \tilde{Z}_{s}^{2} d s}\right] \leq$ $\|\tilde{Y}\|_{\mathcal{S}^{2}}\|\tilde{Z}\|_{2 \beta}<\infty$. By standard arguments, we deduce $E\left[\int_{0}^{T} \mathrm{e}^{\beta s} \tilde{Y}_{s-} \tilde{Z}_{s} d W_{s}\right]=0$. By similar arguments, the last term on the r.h.s. of inequality (3.8) has also zero expectation.

By applying (3.8) with $t=0$, and by taking expectations on both sides of the resulting inequality, we obtain $\tilde{Y}_{0}^{2}+\|\tilde{Z}\|_{\beta}^{2}+\|\tilde{k}\|_{\nu, \beta}^{2} \leq \varepsilon^{2}\|\tilde{f}\|_{\beta}^{2}$. We deduce the desired estimates:

$$
\|\tilde{Z}\|_{\beta}^{2} \leq \varepsilon^{2}\|\tilde{f}\|_{\beta}^{2} \text { and }\|\tilde{k}\|_{\nu, \beta}^{2} \leq \varepsilon^{2}\|\tilde{f}\|_{\beta}^{2} .
$$

Estimate for $\|\tilde{Y}\|_{\beta}^{2}$. From inequality (3.7) we derive that a.s., for all $t \in[0, T]$,

$$
\mathrm{e}^{\beta t} \tilde{Y}_{t}^{2} \leq \varepsilon^{2} \int_{] t, T]} \mathrm{e}^{\beta s} \tilde{f}^{2}(s) d s-2 \int_{] t, T]} \mathrm{e}^{\beta s} \tilde{Y}_{s-} \tilde{Z}_{s} d W_{s}-2 \int_{] t, T]} \mathrm{e}^{\beta s} \int_{E} \tilde{Y}_{s-} \tilde{k}_{s}(e) \tilde{N}(d s, d e) .
$$

From this, together with Chasles' relation for stochastic integrals, we get, for all $\tau \in \mathcal{T}_{0, T}$,

$$
\begin{aligned}
\mathrm{e}^{\beta \tau} \tilde{Y}_{\tau}^{2} & \leq \varepsilon^{2} \int_{] 0, T]} \mathrm{e}^{\beta s} \tilde{f}^{2}(s) d s-2 \int_{] 0, T]} \mathrm{e}^{\beta s} \tilde{Y}_{s-} \tilde{Z}_{s} d W_{s}+2 \int_{] 0, \tau]} \mathrm{e}^{\beta s} \tilde{Y}_{s-} \tilde{Z}_{s} d W_{s} \\
& -2 \int_{] 0, T]} \mathrm{e}^{\beta s} \int_{E} \tilde{Y}_{s-} \tilde{k}_{s}(e) \tilde{N}(d s, d e)+2 \int_{] 0, \tau]} \mathrm{e}^{\beta s} \int_{E} \tilde{Y}_{s-} \tilde{k}_{s}(e) \tilde{N}(d s, d e) \text { a.s. }
\end{aligned}
$$

By taking first the essential supremum over $\tau \in \mathcal{T}_{0, T}$ and then the expectation on both sides of the above inequality, we obtain

$\left.\left.\underset{\tau \in \mathcal{T}_{0, T}}{E\left[\operatorname{essup} \mathrm{e}^{\beta \tau}\right.} \tilde{Y}_{\tau}^{2}\right] \leq \varepsilon^{2}\|\tilde{f}\|_{\beta}^{2}+2 E\left[\underset{\tau \in \mathcal{T}_{0, T}}{\operatorname{exssup}}\left|\int_{0}^{\tau} \mathrm{e}^{\beta s} \tilde{Y}_{s-} \tilde{Z}_{s} d W_{s}\right|\right]+\underset{\tau \in \mathcal{T}_{0, T}}{2 E[\operatorname{ess} \sup }\left|\int_{] 0, \tau]} \mathrm{e}^{\beta s} \int_{E} \tilde{Y}_{s-} \tilde{k}_{s}(e) \tilde{N}(d s, d e)\right|\right]$.

Let us consider the last term in (3.12). By using Remark A.14 applied to the right-continuous process $\left(\int_{] 0, t]} \mathrm{e}^{\beta s} \int_{E} \tilde{Y}_{s-} \tilde{k}_{s}(e) \tilde{N}(d s, d e)\right)_{t \in[0, T]}$ and Burkholder-Davis-Gundy inequalities, we get

$$
2 E\left[\underset{\tau \in \mathcal{T}_{0, T}}{\operatorname{ess} \sup }\left|\int_{0}^{\tau} \mathrm{e}^{\beta s} \int_{E} \tilde{Y}_{s-} \tilde{k}_{s}(e) \tilde{N}(d s, d e)\right|\right] \leq 2 c E\left[\sqrt{\int_{0}^{T} \mathrm{e}^{2 \beta s} \int_{E} \tilde{Y}_{s-}^{2} \tilde{k}_{s}^{2}(e) N(d s, d e)}\right],
$$

where $c>0$ is a positive "universal" constant (which does not depend on the other parameters).

The inequality (3.9) and the trivial inequality $a b \leq \frac{1}{2} a^{2}+\frac{1}{2} b^{2}$ lead to 


$$
\begin{array}{r}
2 c E\left[\sqrt{\int_{0}^{T} \mathrm{e}^{2 \beta s} \int_{E} \tilde{Y}_{s-}^{2} \tilde{k}_{s}^{2}(e) N(d s, d e)}\right] \leq E\left[\sqrt{\frac{1}{2} \underset{\tau \in \mathcal{T}_{0, T}}{\operatorname{essupe} \mathrm{e}^{\beta \tau} \tilde{Y}_{\tau}^{2}} \sqrt{8 c^{2} \int_{0}^{T} \mathrm{e}^{\beta s} \int_{E} \tilde{k}_{s}^{2}(e) N(d s, d e)}}\right. \\
\leq \frac{1}{4} E\left[\underset{\tau \in \mathcal{T}_{0, T}}{\operatorname{ess} \sup ^{\beta \tau}} \tilde{Y}_{\tau}^{2}\right]+4 c^{2} E\left[\int_{0}^{T} \mathrm{e}^{\beta s} \int_{E} \tilde{k}_{s}^{2}(e) N(d s, d e)\right]=\frac{1}{4}\|\tilde{Y}\|_{\beta}^{2}+4 c^{2}\|\tilde{k}\|_{\nu, \beta}^{2} .
\end{array}
$$

Here, the equality has been obtained by adding and subtracting $4 c^{2}\|\tilde{k}\|_{\nu, \beta}^{2}$ (on the lefthand side) and by using the fact that $E\left[\int_{0}^{T} \mathrm{e}^{\beta s} \int_{E} \tilde{k}_{s}^{2}(e) \tilde{N}(d s, d e)\right]=0$. By using similar arguments, we obtain that the last but one term in (3.12) satisfies

$$
\left.\underset{\tau \in \mathcal{T}_{0, T}}{2 E\left[\operatorname{ess} \sup _{0}^{\tau}\right.} \int^{\beta s} \tilde{Y}_{s-} \tilde{Z}_{s} d W_{s}\right] \leq \frac{1}{2}\|\tilde{Y}\|_{\beta}^{2}+2 c^{2}\|\tilde{Z}\|_{\beta}^{2},
$$

where $c$ is the same universal constant as above. By (3.12), we thus derive that $\frac{1}{4}\|\tilde{Y}\|_{\beta}^{2} \leq$ $\varepsilon^{2}\|\tilde{f}\|_{\beta}^{2}+2 c^{2}\|\tilde{Z}\|_{\beta}^{2}+4 c^{2}\|\tilde{k}\|_{\nu, \beta}^{2}$. This inequality, together with the estimates from (3.10), gives $\|\tilde{Y}\|_{\beta}^{2} \leq 4 \varepsilon^{2}\left(1+6 c^{2}\right)\|\tilde{f}\|_{\beta}^{2}$, which is the desired result.

In the following lemma, we prove existence and uniqueness of the solution to the RBSDE from Definition 3.1 (in the case where the driver $f$ does not depend on $y, z$ and $k$ ) and we characterize the first component of the solution as the "value process" of an optimal stopping problem.

Lemma 3.3 Suppose that $f$ does not depend on $y, z, \mathcal{K}$, that is $f(\omega, t, y, z, \mathcal{K})=f(\omega, t)$, where $f$ is a progressive process with $E\left[\int_{0}^{T} f(t)^{2} d t\right]<+\infty$. Let $\left(\xi_{t}\right)$ be an obstacle. Then, the RBSDE from Definition 3.1 admits a unique solution $(Y, Z, k, A, C) \in \mathcal{S}^{2} \times \mathbb{H}^{2} \times \mathbb{H}_{\nu}^{2} \times$ $\mathcal{S}^{2} \times \mathcal{S}^{2}$, and for each $S \in \mathcal{T}_{0, T}$, we have

$$
Y_{S}=\underset{\tau \in \mathcal{T}_{S, T}}{\operatorname{essup} \sup } E\left[\xi_{\tau}+\int_{S}^{\tau} f(t) d t \mid \mathcal{F}_{S}\right] \quad \text { a.s. }
$$

Moreover, the following property holds:

$$
Y_{S}=\xi_{S} \vee Y_{S+} \text { a.s. }
$$

We also have $Y_{S+}=\operatorname{ess}_{\sup _{\tau>S}} E\left[\xi_{\tau}+\int_{S}^{\tau} f(t) d t \mid \mathcal{F}_{S}\right] \quad$ a.s., for all $S \in \mathcal{T}_{0, T}$. If, furthermore, the obstacle $\left(\xi_{t}\right)$ is l.u.s.c. along stopping times, then $\left(A_{t}\right)$ is continuous.

The proof of the lemma is divided in several steps. First, we exhibit a "natural candidate" $\bar{Y}$ to be the first component of the solution to the RBSDE with parameters $(f, \xi)$; we prove 
that $\bar{Y}$ belongs to the space $\mathcal{S}^{2}$ and we give an estimate of $\|\mid \bar{Y}\| \|_{\mathcal{S}^{2}}^{2}$ in terms of $\|\xi\|_{\mathcal{S}^{2}}^{2}$ and $\|f\|_{H^{2}}^{2}$. In the second step, we exhibit "natural candidates" for the processes $A$ and $C$, and a "natural candidate" $M$ for the martingale part of the solution to the RBSDE with parameters $(f, \xi)$. In the third step, we prove that the processes $A$ and $C$ belong to $\mathcal{S}^{2}$ and we give an estimate of $\left\|A+C_{-}\right\|_{\mathcal{S}^{2}}^{2}$. In the fourth step, we apply the martingale representation theorem to $M$, which gives the second component $Z \in \mathbb{H}^{2}$ and the third component $k \in \mathbb{H}_{\nu}^{2}$ of the solution. In the fifth step, we show the uniqueness of the solution. Finally, we prove property (3.16) and the last two assertions of the lemma.

Proof: For $S \in \mathcal{T}_{0, T}$, we define $\bar{Y}(S)$ by

$$
\bar{Y}(S):=\operatorname{esssup}_{\tau \in \mathcal{T}_{S, T}} E\left[\xi_{\tau}+\int_{S}^{\tau} f(u) d u \mid \mathcal{F}_{S}\right] .
$$

By Proposition A.6 in the Appendix, there exists a ladlag optional process $\left(\bar{Y}_{t}\right)_{t \in[0, T]}$ which aggregates the family $(\bar{Y}(S))_{S \in \mathcal{T}_{0, T}}$, that is,

$$
\bar{Y}_{S}=\bar{Y}(S) \text { a.s. for all } S \in \mathcal{T}_{0, T} .
$$

Step 1. By using Jensen's inequality and the triangular inequality, we get

$$
\left.\left|\bar{Y}_{S}\right| \leq \operatorname{esssup}_{\tau \in \mathcal{T}_{S, T}}^{\operatorname{ess}} E\left[\left|\xi_{\tau}\right|+\left|\int_{S}^{\tau} f(u) d u\right| \mid \mathcal{F}_{S}\right] \leq \underset{\tau \in \mathcal{T}_{S, T}}{E\left[\operatorname{esssup}_{\tau}\right.}\left|\xi_{\tau}\right|+\int_{0}^{T}|f(u)| d u \mid \mathcal{F}_{S}\right]=E\left[X \mid \mathcal{F}_{S}\right]
$$

a.s., for all $S \in \mathcal{T}_{0, T}$, where we have set

$$
X:=\int_{0}^{T}|f(u)| d u+\underset{\tau \in \mathcal{T}_{0, T}}{\operatorname{ess} \sup }\left|\xi_{\tau}\right| .
$$

We apply Cauchy-Schwarz inequality to obtain

$$
E\left[X^{2}\right] \leq c T\|f\|_{H^{2}}^{2}+c\|\| \xi \|_{\mathcal{S}^{2}}^{2},
$$

where $c>0$ is a positive constant, which, in the sequel, is allowed to differ from line to line.

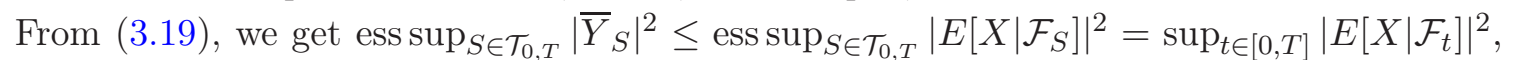
where the equality follows from the right-continuity of the process $\left(E\left[X \mid \mathcal{F}_{t}\right]\right)_{0 \leq t \leq T}$, together with Remark A.14, By using this and Doob's martingale inequalities in $L^{2}$, we obtain

$$
E\left[\underset{S \in \mathcal{T}_{0, T}}{\operatorname{ess} \sup _{S}}\left|\bar{Y}_{S}\right|^{2}\right] \leq E\left[\sup _{t \in[0, T]}\left|E\left[X \mid \mathcal{F}_{t}\right]\right|^{2}\right] \leq c E\left[X^{2}\right] \leq c T\|f\|_{H^{2}}^{2}+c\|\xi\|_{\mathcal{S}^{2}}^{2}
$$

where the last inequality follows from (3.21). 
Step 2. By Proposition A.6, the process $\left(\bar{Y}_{t}+\int_{0}^{t} f(u) d u\right)_{t \in[0, T]}$ is a strong supermartingale. Due to the previous step and to the assumption $f \in \mathbb{H}^{2}$, it is of class (D). Applying Mertens decomposition (cf. Theorem A.1) gives the following

$$
\bar{Y}_{t}=-\int_{0}^{t} f(u) d u+M_{t}-A_{t}-C_{t-} \text { for all } t \in[0, T] \text { a.s., }
$$

where $M$ is a cadlag uniformly integrable martingale, $A$ is a nondecreasing right-continuous predictable process such that $A_{0}=0, E\left(A_{T}\right)<\infty$, and $C$ is a nondecreasing rightcontinuous adapted purely discontinuous process such that $C_{0-}=0, E\left(C_{T}\right)<\infty$. Let $\tau \in \mathcal{T}_{0, T}$. By Remark A.17, $\Delta_{+} \bar{Y}_{\tau}=\mathbf{1}_{\left\{\bar{Y}_{\tau}=\xi_{\tau}\right\}} \Delta_{+} \bar{Y}_{\tau}$ a.s. Now, by $(3.23), \Delta C_{\tau}=-\Delta_{+} \bar{Y}_{\tau}$ a.s. It follows that $\Delta C_{\tau}=\mathbf{1}_{\left\{\bar{Y}_{\tau}=\xi_{\tau}\right\}} \Delta C_{\tau}$ a.s. In other terms, the process $C$ satisfies the minimality condition (3.4) (with $Y$ replaced by $\bar{Y}$ ). Moreover, thanks to a result from optimal stopping theory due to El Karoui (cf. [11, Prop. 2.34]; cf. also [22]), for each predictable stopping time $\tau$, we have $\Delta A_{\tau}=\mathbf{1}_{\left\{\bar{Y}_{\tau-}=\xi_{\tau-}\right\}} \Delta A_{\tau}$ a.s. For the continuous part $A^{c}$ of $A$, again by a result from optimal stopping theory (cf. [23]), we have $\int_{0}^{T} \mathbf{1}_{\left\{\bar{Y}_{t}>\xi_{t}\right\}} d A_{t}^{c}=0$ a.s. The process $A$ thus satisfies the minimality condition (3.3) (with $Y$ replaced by $\bar{Y}$ ). We have $\bar{Y}_{T}=\bar{Y}(T)=\xi_{T}$ a.s. (due to (3.17) and (3.18)). Also, from (3.17) and (3.18), we have $\bar{Y}_{S}=\bar{Y}(S) \geq \xi_{S}$ a.s. for all $S \in \mathcal{T}_{0, T}$, which, along with a classical result of the general theory of processes (cf. [8, Theorem IV.84]) implies that $\bar{Y}_{t} \geq \xi_{t}, 0 \leq t \leq T$, a.s.

Step 3. Let us consider the Mertens process associated with the strong supermartingale $\bar{Y} .+\int_{0} f(u) d u$, that is the process $\left(A_{t}+C_{t-}\right)$, where the processes $\left(A_{t}\right)$ and $\left(C_{t-}\right)$ are given by (3.23). We show that $A_{T}+C_{T-} \in L^{2}$. By arguments similar to those used in the proof of (3.19), we see that $\left|\bar{Y}_{S}+\int_{0}^{S} f(u) d u\right| \leq E\left[X \mid \mathcal{F}_{S}\right]$, where $X$ is the random variable defined in (3.20). This observation, together with a result from potential theory (cf. Corollary A.1), gives $E\left[\left(A_{T}+C_{T-}\right)^{2}\right] \leq c E\left[X^{2}\right]$, where $c>0$. By combining this inequality with inequality (3.21), we obtain

$$
E\left[\left(A_{T}+C_{T-}\right)^{2}\right] \leq c T\|f\|_{H^{2}}^{2}+c\|\| \xi \|_{\mathcal{S}^{2}}^{2},
$$

where we have again allowed the positive constant $c$ to vary from line to line. We conlude that $A_{T}+C_{T-} \in L^{2}$. Hence, $A_{T}$ and $C_{T-}\left(=C_{T}\right)$ are square integrable, which, due to the nondecreasingness of $A$ and $C$, is equivalent to $A \in \mathcal{S}^{2}$ and $C \in \mathcal{S}^{2}$.

Step 4. The martingale $M$ from the decomposition (3.23) belongs to $\mathcal{S}^{2}$; this is a consequence of Step 1., Step 3., and the fact that the process $\left(\int_{0}^{t} f(u) d u\right)_{t \in[0, T]}$ is in $\mathcal{S}^{2}$ (since $f \in \mathbb{H}^{2}$ ). By the martingale representation theorem (cf., e.g., Lemma 2.3 in [37]) there exists a unique predictable process $Z \in \mathbb{H}^{2}$ and a unique predictable $k \in \mathbb{H}_{\nu}^{2}$ such that $d M_{t}=Z_{t} d W_{t}+\int_{E} k_{t}(e) \tilde{N}(d t, d e)$. Combining this step with the previous ones gives that $(\bar{Y}, Z, k, A, C)$ is a solution to the RBSDE with parameters $f$ and $\xi$. 
Step 5. Let us now prove the uniqueness of the solution. Let $Y$ be the first component of a solution to the RBSDE with driver $f$ and obstacle $\xi$. Then, by the previous Lemma 3.2 (applied with $f^{1}=f^{2}=f$ ) we obtain $Y=\bar{Y}$ in $\mathcal{S}^{2}$, where $\bar{Y}$ is given by (3.17). The uniqueness of the other components follows from the uniqueness of Mertens decomposition of strong optional supermartingales and from the uniqueness of the martingale representation. (We note that the uniqueness of the second and the third component can be obtained also by applying the previous Lemma 3.2.)

Step 6. Property (3.16) and the characterization of $Y_{S+}$ as the value function of an optimal stopping problem follow from Proposition A.6 parts (ii) and (iii). The last assertion of Lemma 3.3 follows from classical results (cf., for instance, the last statement in Thm. 20 of [9, page 429], or [22]).

With the help of the previous two lemmas, we now prove the existence and uniqueness of the solution to the RBSDE from Definition 3.1 in the case of a general Lipschitz driver.

Theorem 3.4 (Existence and uniqueness of the solution) Let $\xi$ be a left-limited and r.u.s.c. process in $\mathcal{S}^{2}$ and let $f$ be a Lipschitz driver. The RBSDE with parameters $(f, \xi)$ from Definition 3.1 admits a unique solution $(Y, Z, k, A, C) \in \mathcal{S}^{2} \times \mathbb{H}^{2} \times \mathbb{H}_{\nu}^{2} \times \mathcal{S}^{2} \times \mathcal{S}^{2}$. Moreover, for all $S \in \mathcal{T}_{0, T}$, we have

$$
Y_{S}=\xi_{S} \vee Y_{S+} \text { a.s. }
$$

Furthermore, if $\left(\xi_{t}\right)$ is assumed l.u.s.c. along stopping times, then $\left(A_{t}\right)$ is continuous (or equivalently, the process $\left(Y_{t}\right)$ is l.u.s.c. along stopping times).

Remark 3.6 We will see that, as in the right-continuous case, the existence and uniqueness result follows from a fixed point theorem applied in an appropriate Banach space. In the right-continuous case, the Banach space is classically the product space $\mathbb{H}^{2} \times \mathbb{H}^{2} \times \mathbb{H}_{\nu}^{2}$ equipped with the norm $\|Y\|_{\beta}^{2}+\|Z\|_{\beta}^{2}+\|k\|_{\nu, \beta}^{2}$ (cf, e.g. [12], [20], [34]). However, this Banach space does not suit our purpose. Indeed, let us make the following observation. Let $Y$ be an optional process such that $\|Y\|_{\beta}=0$. We then have $Y_{t}=0,0 \leq t \leq T d P \otimes d t$-a.e. When $Y$ is right-continuous, this implies the indistinguishability of $Y$ from the null process 0 , that is, the property $Y_{t}=0,0 \leq t \leq T$ a.s. However, if $Y$ is not right-continuous, the implication is not necessarily true. ${ }^{2}$ Hence, applying a fixed point theorem in this space cannot give us uniqueness of the solution of our reflected BSDE in the sense of processes, that is, up to indistinguishability.

\footnotetext{
${ }^{2}$ However, the property holds for the "triple bar" map $\|\cdot\| \|_{\beta}$ on $\mathcal{S}^{2}$. More precisely, if $Y \in \mathcal{S}^{2}$ with $\|Y\|_{\beta}=0$, then $Y_{t}=0,0 \leq t \leq T$ a.s. because $\|\cdot\|_{\beta}$ is a norm on $\mathcal{S}^{2}$. Note that $\|\cdot\|_{\beta}$ is only a semi-norm on $\mathcal{S}^{2}$.
} 
Proof: For each $\beta>0$, we denote by $\mathcal{B}_{\beta}^{2}$ the space $\mathcal{S}^{2} \times \mathbb{H}^{2} \times \mathbb{H}_{\nu}^{2}$ which we equip with the norm $\|(\cdot, \cdot, \cdot)\|_{\mathcal{B}_{\beta}^{2}}$ defined by $\|(Y, Z, k)\|_{\mathcal{B}_{\beta}^{2}}^{2}:=\|Y\|_{\beta}^{2}+\|Z\|_{\beta}^{2}+\|k\|_{\nu, \beta}^{2}$, for $(Y, Z, k) \in$ $\mathcal{S}^{2} \times \mathbb{H}^{2} \times \mathbb{H}_{\nu}^{2}$. Since $\left(\mathbb{H}^{2},\|\cdot\|_{\beta}\right)$ and $\left(\mathbb{H}_{\nu}^{2},\|\cdot\|_{\nu, \beta}\right)$ are Banach spaces, and since by Proposition 2.1, $\left(\mathcal{S}^{2},\|\cdot\|_{\beta}\right)$ is a Banach space, it follows that $\left(\mathcal{B}_{\beta}^{2},\|\cdot\|_{\mathcal{B}_{\beta}}\right)$ is a Banach space.

We define a mapping $\Phi$ from $\mathcal{B}_{\beta}^{2}$ into itself as follows: for a given $(y, z, l) \in \mathcal{B}_{\beta}^{2}$, we set $\Phi(y, z, l):=(Y, Z, k)$, where $Y, Z, k$ are the first three components of the solution $(Y, Z, k, A, C)$ to the RBSDE associated with driver $f(s):=f\left(s, y_{s}, z_{s}, l_{s}\right)$ and with obstacle $\xi$. The mapping $\Phi$ is well-defined by Lemma 3.3.

Let $\left(y^{\prime}, z^{\prime}, l^{\prime}\right)$ and $\left(y^{\prime \prime}, z^{\prime \prime}, l^{\prime \prime}\right)$ be two elements of $\mathcal{B}_{\beta}^{2}$. We set $\left(Y^{\prime}, Z^{\prime}, k^{\prime}\right)=\Phi\left(y^{\prime}, z^{\prime}, l^{\prime}\right)$ and $\left(Y^{\prime \prime}, Z^{\prime \prime}, k^{\prime \prime}\right)=\Phi\left(y^{\prime \prime}, z^{\prime \prime}, l^{\prime \prime}\right)$. We also set $\tilde{Y}:=Y^{\prime}-Y^{\prime \prime}, \tilde{Z}:=Z^{\prime}-Z^{\prime \prime}, \tilde{k}:=k^{\prime}-k^{\prime \prime}$, $\tilde{y}:=y^{\prime}-y^{\prime \prime}, \tilde{z}:=z^{\prime}-z^{\prime \prime}, \tilde{l}:=l^{\prime}-l^{\prime \prime}$.

Let us prove that for a suitable choice of the parameter $\beta>0$ the mapping $\Phi$ is a contraction from the Banach space $\mathcal{B}_{\beta}^{2}$ into itself. By applying Lemma 3.2, we get

$$
\|\tilde{Y}\|_{\beta}^{2}+\|\tilde{Z}\|_{\beta}^{2}+\|\tilde{k}\|_{\nu, \beta}^{2} \leq 6 \varepsilon^{2}\left(1+4 c^{2}\right)\left\|f\left(y^{\prime}, z^{\prime}, l^{\prime}\right)-f\left(y^{\prime \prime}, z^{\prime \prime}, l^{\prime \prime}\right)\right\|_{\beta}^{2},
$$

for all $\varepsilon>0$, for all $\beta \geq \frac{1}{\varepsilon^{2}}$. By using the Lipschitz property of $f$ and the fact that $(a+b+c)^{2} \leq 3\left(a^{2}+b^{2}+c^{2}\right)$ for all $(a, b, c) \in \mathbf{R}^{3}$, we obtain $\left\|f\left(y^{\prime}, z^{\prime}, l^{\prime}\right)-f\left(y^{\prime \prime}, z^{\prime \prime}, l^{\prime \prime}\right)\right\|_{\beta}^{2} \leq$ $C_{K}\left(\|\tilde{y}\|_{\beta}^{2}+\|\tilde{z}\|_{\beta}^{2}+\|\tilde{l}\|_{\nu, \beta}^{2}\right)$, where $C_{K}$ is a positive constant depending on the Lipschitz constant $K$ only. Thus, for all $\varepsilon>0$, for all $\beta \geq \frac{1}{\varepsilon^{2}}$, we have $\|\tilde{Y}\|_{\beta}^{2}+\|\tilde{Z}\|_{\beta}^{2}+\|\tilde{k}\|_{\nu, \beta}^{2} \leq$ $6 \varepsilon^{2} C_{K}\left(1+4 c^{2}\right)\left(\|\tilde{y}\|_{\beta}^{2}+\|\tilde{z}\|_{\beta}^{2}+\|\tilde{l}\|_{\nu, \beta}^{2}\right)$. Now, using Fubini's theorem, we get $\|\tilde{y}\|_{\beta}^{2} \leq T\|\tilde{y}\|_{\beta}^{2}$. Hence, we have

$$
\|\tilde{Y}\|_{\beta}^{2}+\|\tilde{Z}\|_{\beta}^{2}+\|\tilde{k}\|_{\nu, \beta}^{2} \leq 6 \varepsilon^{2} C_{K}\left(1+4 c^{2}\right)(T+1)\left(\|\tilde{y}\|_{\beta}^{2}+\|\tilde{z}\|_{\beta}^{2}+\|\tilde{l}\|_{\nu, \beta}^{2}\right) .
$$

Thus, for $\varepsilon>0$ such that $6 \varepsilon^{2} C_{K}\left(1+4 c^{2}\right)(T+1)<1$ and $\beta>0$ such that $\beta \geq \frac{1}{\varepsilon^{2}}$ the mapping $\Phi$ is a contraction. By the Banach fixed-point theorem, we get that $\Phi$ has a unique fixed point in $\mathcal{B}_{\beta}^{2}$, denoted by $(Y, Z, k)$, that is, such that $(Y, Z, k)=\Phi(Y, Z, k)$. By definition of the mapping $\Phi$, the process $(Y, Z, k)$ is thus equal to the first three components of the solution $(Y, Z, k, A, C)$ to the reflected BSDE associated with the driver process $g(\omega, t):=f\left(\omega, t, Y_{t}(\omega), Z_{t}(\omega), k_{t}(\omega)\right)$ and with obstacle $\xi$. It follows that $(Y, Z, k, A, C)$ is the unique solution to the RBSDE with parameters $(f, \xi)$.

Property (3.25) follows from Eq. (3.16) of Lemma 3.3 and from the fact that $(Y, Z, k, A, C)$ is equal to the solution of the reflected BSDE associated with the driver process $g(\omega, t):=$ $f\left(\omega, t, Y_{t}(\omega), Z_{t}(\omega), k_{t}(\omega)\right)$.

The last assertion of the theorem follows from Lemma 3.3 (fourth assertion) applied with the process $g(\omega, t):=f\left(\omega, t, Y_{t}(\omega), Z_{t}(\omega), k_{t}(\omega)\right)$. 


\section{Optimal stopping with $f$-conditional expectations.}

4.1. Formulation of the problem. Let $T>0$ be the terminal time and $f$ be a predictable Lipschitz driver. Let $\left(\xi_{t}, 0 \leq t \leq T\right)$ be a left-limited r.u.s.c. process in $\mathcal{S}^{2}$ modelling a dynamic financial position. The risk of $\xi$ is assessed by a dynamic risk measure equal, up to a minus sign, to the $f$-conditional expectation of $\xi$. More precisely: let $T^{\prime} \in[0, T]$ be a fixed (for the present) instant before the terminal time $T$; the gain of the position at $T^{\prime}$ is equal to $\xi_{T^{\prime}}$ and the risk at time $t$, where $t \in\left[0, T^{\prime}\right]$, is assessed by $-\mathcal{E}_{t, T^{\prime}}^{f}\left(\xi_{T^{\prime}}\right)$. Here, we use the usual notation $\mathcal{E}_{\cdot, T^{\prime}}^{f}\left(\xi_{T^{\prime}}\right)$ for the first component of the BSDE with driver $f$, terminal time $T^{\prime}$ and terminal condition $\xi_{T^{\prime}}$; the random variable $\mathcal{E}_{t, T^{\prime}}^{f}\left(\xi_{T^{\prime}}\right)$ is referred to as the $f$-conditional expectation of $\xi_{T^{\prime}}$ at time $t$. The modelling is similar when $T^{\prime} \in[0, T]$ is replaced by a more general stopping time $\tau \in \mathcal{T}_{0, T}{ }^{3}$.

We are now interested in stopping the process $\xi$ in such a way that the risk be minimal. We are thus led to formulating the following optimal stopping problem (at time 0):

$$
v(0)=-\operatorname{ess} \sup _{\tau \in \mathcal{T}_{0, T}} \mathcal{E}_{0, \tau}^{f}\left(\xi_{\tau}\right) \text {. }
$$

We recall that in our framework (as opposed to the simpler case of a brownian filtration) the monotonicity property of $f$-conditional expectations is not automatically satisfied. From now on we make the following assumption on the driver $f$, which ensures the nondecreasing property of $\mathcal{E}^{f}(\cdot)$ by the comparison theorem for BSDEs with jumps (cf. [33, Thm. 4.2]).

Assumption 4.1 Assume that $d P \otimes d t$-a.e. for each $\left(y, z, k_{1}, k_{2}\right) \in \mathbb{R}^{2} \times\left(L_{\nu}^{2}\right)^{2}$,

$$
f\left(t, y, z, k_{1}\right)-f\left(t, y, z, \kappa_{2}\right) \geq\left\langle\theta_{t}^{y, z, \kappa_{1}, \kappa_{2}}, \kappa_{1}-\kappa_{2}\right\rangle_{\nu}
$$

with

$$
\theta:[0, T] \times \Omega \times \mathbb{R}^{2} \times\left(L_{\nu}^{2}\right)^{2} \rightarrow L_{\nu}^{2} ;\left(\omega, t, y, z, k_{1}, k_{2}\right) \mapsto \theta_{t}^{y, z, k_{1}, k_{2}}(\omega, \cdot)
$$

$\mathcal{P} \otimes \mathcal{B}\left(\mathbf{R}^{2}\right) \otimes \mathcal{B}\left(\left(L_{\nu}^{2}\right)^{2}\right)$-measurable, satisfying $\left\|\theta_{t}^{y, z, k_{1}, \kappa_{2}}(\cdot)\right\|_{\nu} \leq K$ for all $\left(y, z, \kappa_{1}, \kappa_{2}\right) \in$ $\mathbb{R}^{2} \times\left(L_{\nu}^{2}\right)^{2}, d P \otimes d t$-a.e., where $K$ is a positive constant, and such that

$$
\theta_{t}^{y, z, k_{1}, k_{2}}(e) \geq-1
$$

for $\operatorname{all}\left(y, z, k_{1}, k_{2}\right) \in \mathbb{R}^{2} \times\left(L_{\nu}^{2}\right)^{2}, d P \otimes d t \otimes d \nu(e)-$ a.e.

\footnotetext{
${ }^{3}$ Recall that a process $Y$ is the solution to the BSDE associated with driver $f$, terminal time $\tau$ and terminal condition $\zeta$ (where $\zeta$ is an $\mathcal{F}_{\tau}$-measurable square-integrable random variable) if for almost all $\omega \in \Omega$, for all $t \in[0, T], Y_{t}(\omega)=\bar{Y}_{t}(\omega)$, where $\bar{Y}$ denotes the solution to the BSDE associated with driver $f \mathbf{1}_{t \leq \tau}$, terminal time $T$ and terminal condition $\zeta$. The process $Y$ is also denoted $\mathcal{E}_{,, \tau}^{f}(\zeta)$.
} 
The above assumption is satisfied if, for example, $f$ is of class $\mathcal{C}^{1}$ with respect to $k$ such that $\nabla_{k} f$ is bounded (in $L_{\nu}^{2}$ ) and $\nabla_{k} f \geq-1$ (see Proposition A.2. in [10]).

Remark 4.7 The strict comparison theorem for BSDEs with jumps (cf. Theorem 4.4 in [33]) ensures that if the inequality (4.27) is strict, then $\mathcal{E}^{f}(\cdot)$ is strictly monotonous in the following sense: for $\tau \in \mathcal{T}_{0, T}$, for $\xi^{1}, \xi^{2} \in L^{2}\left(\mathcal{F}_{\tau}\right)$ such that $\xi^{1} \leq \xi^{2}$ a.s., and for $S \in \mathcal{T}_{0, T}$ such that $S \leq \tau$ a.s., the property $\mathcal{E}_{S, \tau}^{f}\left(\xi^{1}\right)=\mathcal{E}_{S, \tau}^{f}\left(\xi^{2}\right)$ a.s., implies $\xi^{1}=\xi^{2}$ a.s.

A counter-example to the strict monotonicity of $\mathcal{E}^{f}(\cdot)$ in the case where the strict inequality in (4.27) is not assumed is given in [33] (cf. also Example A.2 in the Appendix).

As is usual in optimal control, we embed the above problem (4.26) in a larger class of problems. We thus consider for each $S \in \mathcal{T}_{0, T}$, the random variable

$$
v(S)=-\operatorname{ess} \sup _{\tau \in \mathcal{T}_{S, T}} \mathcal{E}_{S, \tau}^{f}\left(\xi_{\tau}\right)
$$

which corresponds to the minimal risk measure at time $S$. Our aim is to characterize $v(S)$ for each $S \in \mathcal{T}_{0, T}$, and to study the existence of an $S$-optimal stopping time $\tau^{*} \in \mathcal{T}_{S, T}$, i.e. a stopping time $\tau^{*} \in \mathcal{T}_{S, T}$ such that $v(S)=-\mathcal{E}_{S, \tau^{*}}^{f}\left(\xi_{\tau^{*}}\right)$ a.s.

4.2. Characterization of the value function as the solution of an RBSDE. In this section, we show that the minimal risk measure $v$ defined by (4.28) coincides with $-Y$, where $Y$ is (the first component of) the solution to the reflected BSDE associated with driver $f$ and obstacle $\xi$. We also investigate the question of the existence of an $\varepsilon$-optimal stopping time, and that of the existence of an optimal stopping time (under suitable assumptions on the process $\xi$ ).

The following terminology will be used in the sequel. Let $Y$ be a process in $\mathcal{S}^{2}$. Let $f$ be a predictable Lipschitz driver satisfying Assumption 4.1.

- The process $\left(Y_{t}\right)$ is said to be a strong $\mathcal{E}^{f}$-supermartingale (resp $\mathcal{E}^{f}$-submartingale), if $\mathcal{E}_{S, \tau}^{f}\left(Y_{\tau}\right) \leq Y_{S}\left(\operatorname{resp} . \mathcal{E}_{S, \tau}^{f}\left(Y_{\tau}\right) \geq Y_{S}\right)$ a.s. on $S \leq \tau$, for all $S, \tau \in \mathcal{T}_{0, T}$.

The process $\left(Y_{t}\right)$ is said to be a strong $\mathcal{E}^{f}$-martingale if it is both a strong $\mathcal{E}^{f}$-super and $\mathcal{E}^{f}$-submartingale.

- Let $S, \tau \in \mathcal{T}_{0, T}$ be such that $S \leq \tau$ a.s. The process $Y$ is said to be a strong $\mathcal{E}^{f}$ supermartingale (resp. a strong $\mathcal{E}^{f}$-submartingale) on $[S, \tau]$ if for all $\sigma, \mu \in \mathcal{T}_{0, T}$ such that $S \leq \sigma \leq \mu \leq \tau$ a.s., we have $Y_{\sigma} \geq \mathcal{E}_{\sigma, \mu}^{f}\left(Y_{\mu}\right)$ a.s. (resp. $Y_{\sigma} \leq \mathcal{E}_{\sigma, \mu}^{f}\left(Y_{\mu}\right)$ a.s.) We say that $Y$ is a strong $\mathcal{E}^{f}$-martingale on $[S, \tau]$ if it is both a strong $\mathcal{E}^{f}$-super and submartingale on $[S, \tau]$.

Remark 4.8 We note that a process $Y \in \mathcal{S}^{2}$ is a strong $\mathcal{E}^{f}$-martingale on $[S, \tau]$ (where $S$, $\tau \in \mathcal{T}_{0, T}$ are such that $S \leq \tau$ a.s.) if and only if, on $[S, \tau], Y$ is indistinguishable from the 
solution to the BSDE associated with driver $f$, terminal time $\tau$ and terminal condition $Y_{\tau}$. It follows that for a process $Y \in \mathcal{S}^{2}$ to be a strong $\mathcal{E}^{f}$-martingale on $[S, \tau]$, it is sufficient to have: $Y_{\sigma}=\mathcal{E}_{\sigma, \tau}^{f}\left(Y_{\tau}\right)$ a.s., for all $\sigma \in \mathcal{T}_{0, T}$ such that $S \leq \sigma \leq \tau$ a.s.

Property 4.1 Let $f$ be a predictable Lipschitz driver satisfying Assumption 4.1. Let $S, \tau \in$ $\mathcal{T}_{0, T}$ with $S \leq \tau$ a.s. Let $Y$ be a strong $\mathcal{E}^{f}$-supermartingale on $[S, \tau]$. We introduce the following two assertions:

(i) The process $Y$ is a strong $\mathcal{E}^{f}$-martingale on $[S, \tau]$.

(ii) $Y_{S}=\mathcal{E}_{S, \tau}^{f}\left(Y_{\tau}\right)$ a.s.

Assertion (i) implies Assertion (ii).

If, in Assumption 4.1, we further assume the strict inequality $\theta_{t}^{y, z, \kappa_{1}, \kappa_{2}}>-1$, then Assertion (ii) implies Assertion (i).

Proof: The implication $(i) \Rightarrow(i i)$ is due to the definition. Let us show the converse implication. Let $\sigma \in \mathcal{T}_{0, T}$ be such that $S \leq \sigma \leq \tau$ a.s. By using (ii) and the consistency property of $f$-expectations, we obtain $Y_{S}=\mathcal{E}_{S, \sigma}^{f}\left(\mathcal{E}_{\sigma, \tau}^{f}\left(Y_{\tau}\right)\right)$ a.s. By using the strong $\mathcal{E}^{f}$-supermartingale property of $Y$ and the monotonicity of $f$-expectations, we obtain $\mathcal{E}_{S, \sigma}^{f}\left(\mathcal{E}_{\sigma, \tau}^{f}\left(Y_{\tau}\right)\right) \leq \mathcal{E}_{S, \sigma}^{f}\left(Y_{\sigma}\right) \leq Y_{S}$ a.s. From the previous two equations we get $Y_{S}=$ $\mathcal{E}_{S, \sigma}^{f}\left(\mathcal{E}_{\sigma, \tau}^{f}\left(Y_{\tau}\right)\right)=\mathcal{E}_{S, \sigma}^{f}\left(Y_{\sigma}\right)$ a.s. In particular,

$$
\mathcal{E}_{S, \sigma}^{f}\left(Y_{\sigma}\right)=\mathcal{E}_{S, \sigma}^{f}\left(\mathcal{E}_{\sigma, \tau}^{f}\left(Y_{\tau}\right)\right) \text { a.s. }
$$

Since $\theta_{t}^{y, z, k_{1}, k_{2}}>-1, \mathcal{E}^{f}(\cdot)$ is strictly monotonous (cf. Remark 4.7). From this, together with equality (4.29) and the inequality $Y_{\sigma} \geq \mathcal{E}_{\sigma, \tau}^{f}\left(Y_{\tau}\right)$ a.s., we get $Y_{\sigma}=\mathcal{E}_{\sigma, \tau}^{f}\left(Y_{\tau}\right)$ a.s. The process $Y$ is thus a strong $\mathcal{E}^{f}$-martingale on $[S, \tau]$.

We next show a lemma which will be used in the proof of the main result of this section.

Lemma 4.1 Let $f$ be a predictable Lipschitz driver satisfying Assumption 4.1 and $\xi$ be a left-limited r.u.s.c. process in $\mathcal{S}^{2}$. Let $(Y, Z, k, A, C)$ be the solution to the reflected BSDE with parameters $(f, \xi)$ as in Definition 3.1. Let $\varepsilon>0$ and $S \in \mathcal{T}_{0, T}$. Let $\tau_{S}^{\varepsilon}$ be defined by

$$
\tau_{S}^{\varepsilon}:=\inf \left\{t \geq S: Y_{t} \leq \xi_{t}+\varepsilon\right\} .
$$

The following two statements hold:

(i) $Y_{\tau_{S}^{\varepsilon}} \leq \xi_{\tau_{S}^{\varepsilon}}+\varepsilon \quad$ a.s.

(ii) The process $Y$ is a strong $\mathcal{E}^{f}$-martingale on $\left[S, \tau_{S}^{\varepsilon}\right]$. 
We note that $\tau_{S}^{\varepsilon}$ defined in (4.30) is a stopping time as the début after $S$ of a progressive set. Note also that $\tau_{S}^{\varepsilon}$ is valued in $[0, T]$ as $Y_{T}=\xi_{T}$ a.s.

Proof: We first prove statement $(i)$. By way of contradiction, we suppose $P\left(Y_{\tau_{S}^{\varepsilon}}>\xi_{\tau_{S}^{\varepsilon}}+\varepsilon\right)>$ 0 . We have $\Delta C_{\tau_{S}^{\varepsilon}}=C_{\tau_{S}^{\varepsilon}}-C_{\left(\tau_{S}^{\varepsilon}\right)-}=0$ on the set $\left\{Y_{\tau_{S}^{\varepsilon}}>\xi_{\tau_{S}^{\varepsilon}}+\varepsilon\right\}$. On the other hand, due to Remark 3.4, $\Delta C_{\tau_{S}^{\varepsilon}}=Y_{\tau_{S}^{\varepsilon}}-Y_{\left(\tau_{S}^{\varepsilon}\right)+}$. Thus, $Y_{\tau_{S}^{\varepsilon}}=Y_{\left(\tau_{S}^{\varepsilon}\right)+}$ on the set $\left\{Y_{\tau_{S}^{\varepsilon}}>\xi_{\tau_{S}^{\varepsilon}}+\varepsilon\right\}$. Hence,

$$
Y_{\left(\tau_{S}^{\varepsilon}\right)+}>\xi_{\tau_{S}^{\varepsilon}}+\varepsilon \text { on the set }\left\{Y_{\tau_{S}^{\varepsilon}}>\xi_{\tau_{S}^{\varepsilon}}+\varepsilon\right\}
$$

We will obtain a contradiction with this statement. Let us fix $\omega \in \Omega$. By definition of $\tau_{S}^{\varepsilon}(\omega)$, there exists a non-increasing sequence $\left(t_{n}\right)=\left(t_{n}(\omega)\right) \downarrow \tau_{S}^{\varepsilon}(\omega)$ such that $Y_{t_{n}}(\omega) \leq \xi_{t_{n}}(\omega)+\varepsilon$, for all $n \in \mathbb{N}$. Hence, $\lim _{\sup _{n \rightarrow \infty}} Y_{t_{n}}(\omega) \leq \limsup _{n \rightarrow \infty} \xi_{t_{n}}(\omega)+\varepsilon$. As the process $\xi$ is r.u.s.c., we have $\limsup _{n \rightarrow \infty} \xi_{t_{n}}(\omega) \leq \xi_{\tau_{S}^{\varepsilon}}(\omega)$. On the other hand, as $\left(t_{n}(\omega)\right) \downarrow \tau_{S}^{\varepsilon}(\omega)$, we have $\lim \sup _{n \rightarrow \infty} Y_{t_{n}}(\omega)=Y_{\left(\tau_{S}^{\varepsilon}\right)+}(\omega)$. Thus, $Y_{\left(\tau_{S}^{\varepsilon}\right)+}(\omega) \leq \xi_{\tau_{S}^{\varepsilon}}(\omega)+\varepsilon$, which is in contradiction with (4.31). We conclude that $Y_{\tau_{S}^{\varepsilon}} \leq \xi_{\tau_{S}^{\varepsilon}}+\varepsilon$ a.s.

Let us now prove statement $(i i)$. By definition of $\tau_{S}^{\varepsilon}$, we have: for a.e. $\omega \in \Omega$, for all $t \in\left[S(\omega), \tau_{S}^{\varepsilon}(\omega)\left[, Y_{t}(\omega)>\xi_{t}(\omega)+\varepsilon\right.\right.$. Hence, for a.e. $\omega \in \Omega$, the function $t \mapsto A_{t}^{c}(\omega)$ is constant on $\left[S(\omega), \tau_{S}^{\varepsilon}(\omega)\right.$ [; by continuity of almost every trajectory of the process $A^{c}, A^{c}(\omega)$ is constant on the closed interval $\left[S(\omega), \tau_{S}^{\varepsilon}(\omega)\right]$, for a.e. $\omega$. Furthermore, for a.e. $\omega \in \Omega$, the function $t \mapsto A_{t}^{d}(\omega)$ is constant on $\left[S(\omega), \tau_{S}^{\varepsilon}(\omega)\right.$ [. Moreover, $Y_{\left(\tau_{S}^{\varepsilon}\right)-} \geq \xi_{\left(\tau_{S}^{\varepsilon}\right)^{-}}+\varepsilon$ a.s., which implies that $\Delta A_{\tau_{S}^{\varepsilon}}^{d}=0$ a.s. Finally, for a.e. $\omega \in \Omega$, for all $t \in\left[S(\omega), \tau_{S}^{\varepsilon}(\omega)\left[, \Delta C_{t}(\omega)=\right.\right.$ $C_{t}(\omega)-C_{t-}(\omega)=0$; therefore, for a.e. $\omega \in \Omega$, for all $t \in\left[S(\omega), \tau_{S}^{\varepsilon}(\omega)\left[, \Delta_{+} C_{t-}(\omega)=\right.\right.$ $C_{t}(\omega)-C_{t-}(\omega)=0$, which implies that, for a.e. $\omega \in \Omega$, the function $t \mapsto C_{t-}(\omega)$ is constant on $\left[S(\omega), \tau_{S}^{\varepsilon}(\omega)\left[\right.\right.$. By left-continuity of almost every trajectory of the process $\left(C_{t-}\right)$, we get that for a.e. $\omega \in \Omega$, the function $t \mapsto C_{t-}(\omega)$ is constant on the closed interval $\left[S(\omega), \tau_{S}^{\varepsilon}(\omega)\right]$. Thus, for a.e. $\omega \in \Omega$, the map $t \mapsto A_{t}(\omega)+C_{t-}(\omega)$ is constant on $\left[S(\omega), \tau_{S}^{\varepsilon}(\omega)\right]$. Hence, $Y$ is the solution on $\left[S, \tau_{S}^{\varepsilon}\right]$ of the BSDE associated with driver $f$, terminal time $\tau_{S}^{\varepsilon}$ and terminal condition $Y_{\tau_{S}^{\varepsilon}}$. We conclude by using Remark 4.8.

With the help of the previous lemma, we derive the main result of this section.

Theorem 4.2 (Characterization theorem) Let $T>0$ be the terminal time. Let $\left(\xi_{t}, 0 \leq\right.$ $t \leq T$ ) be a left-limited r.u.s.c. process in $\mathcal{S}^{2}$ and let $f$ be a predictable Lipschitz driver satisfying Assumption 4.1. Let $(Y, Z, k, A, C)$ be the solution to the reflected BSDE with parameters $(f, \xi)$ as in Definition 3.1.

(i) For each stopping time $S \in \mathcal{T}_{0}$, we have

$$
Y_{S}=\operatorname{ess} \sup _{\tau \in \mathcal{T}_{S, T}} \mathcal{E}_{S, \tau}^{f}\left(\xi_{\tau}\right) \text { a.s. }
$$


(ii) For each $S \in \mathcal{T}_{0, T}$ and each $\varepsilon>0$, the stopping time $\tau_{S}^{\varepsilon}$ defined by (4.30) is $(L \varepsilon)$ optimal for problem (4.32), that is

$$
Y_{S} \leq \mathcal{E}_{S, \tau_{S}^{\varepsilon}}^{f}\left(\xi_{\tau_{S}^{\varepsilon}}\right)+L \varepsilon \text { a.s. },
$$

where $L$ is a constant which only depends on $T$ and the Lipschitz constant $K$ of $f$.

Remark 4.9 This result still holds when the assumption of existence of left limits for the process $\xi$ is relaxed (cf. also Remark 3.3).

In the case where $\xi$ is right-continuous, we recover Theorem 3.2 of [34].

Proof: Let $\varepsilon>0$ and let $\tau \in \mathcal{T}_{S, T}$. By Proposition A.5 in the Appendix, the process $\left(Y_{t}\right)$ is a strong $\mathcal{E}^{f}$-supermartingale. Hence, for each $\tau \in \mathcal{T}_{S, T}$, we have

$$
Y_{S} \geq \mathcal{E}_{S, \tau}^{f}\left(Y_{\tau}\right) \geq \mathcal{E}_{S, \tau}^{f}\left(\xi_{\tau}\right) \quad \text { a.s. },
$$

where the second inequality follows from the inequality $Y \geq \xi$ and the monotonicity property of $\mathcal{E}^{f}(\cdot)$ (with respect to terminal condition). By taking the supremum over $\tau \in \mathcal{T}_{S, T}$, we get

$$
Y_{S} \geq \operatorname{ess} \sup _{\tau \in \mathcal{T}_{S, T}} \mathcal{E}_{S, \tau}^{f}\left(\xi_{\tau}\right) \quad \text { a.s. }
$$

It remains to show the converse inequality. Due to part $(i i)$ of the previous Lemma 4.1 we have $Y_{S}=\mathcal{E}_{S, \tau_{S}^{\varepsilon}}^{f}\left(Y_{\tau_{S}^{\varepsilon}}\right) \quad$ a.s. From this equality, together with part $(i)$ of Lemma 4.1 and the monotonicity property of $\mathcal{E}^{f}(\cdot)$, we derive

$$
Y_{S}=\mathcal{E}_{S, \tau_{S}^{\varepsilon}}^{f}\left(Y_{\tau_{S}^{\varepsilon}}\right) \leq \mathcal{E}_{S, \tau_{S}^{\varepsilon}}^{f}\left(\xi_{\tau_{S}^{\varepsilon}}+\varepsilon\right) \leq \mathcal{E}_{S, \tau_{S}^{\varepsilon}}^{f}\left(\xi_{\tau_{S}^{\varepsilon}}\right)+L \varepsilon \quad \text { a.s. }
$$

where the last inequality follows from the estimates on BSDEs (cf. Proposition A.4 in [33]). Inequality (4.33) thus holds. From (4.35) we also deduce $Y_{S} \leq \operatorname{ess}_{\sup _{\tau \in \mathcal{T}_{S, T}}} \mathcal{E}_{S, \tau}^{f}\left(\xi_{\tau}\right)+L \varepsilon$ a.s. As $\varepsilon$ is an arbitrary positive number, we get $Y_{S} \leq \operatorname{ess}_{\sup } \operatorname{si}_{S, T} \mathcal{E}_{S, \tau}^{f}\left(\xi_{\tau}\right)$ a.s. By (4.34) this inequality is an equality.

We now investigate the question of the existence of optimal stopping times for the optimal stopping problem (4.32). We first provide an optimality criterion for the problem (4.32).

Proposition 4.2 (Optimality criterion) Let $\left(\xi_{t}, 0 \leq t \leq T\right)$ be a left-limited r.u.s.c. process in $\mathcal{S}^{2}$ and let $f$ be a predictable Lipschitz driver satisfying Assumption 4.1. Let $S \in \mathcal{T}_{0, T}$ and $\hat{\tau} \in \mathcal{T}_{S, T}$. If $Y$ is a strong $\mathcal{E}^{f}$-martingale on $[S, \hat{\tau}]$ with $Y_{\hat{\tau}}=\xi_{\hat{\tau}}$ a.s., then the stopping time $\hat{\tau}$ is $S$-optimal (i.e. $Y_{S}=\mathcal{E}_{S, \hat{\tau}}^{f}\left(\xi_{\hat{\tau}}\right)$ a.s.). The converse statement also holds true, if, in addition, the inequality from Assumption 4.1 is strict (that is, $\theta_{t}^{y, z, k_{1}, k_{2}}>-1$ ). 
Proof: The first claim is immediate. Let us prove the second (and last) claim. Assume the strict inequality in Assumption 4.1. Let $\hat{\tau}$ be $S$-optimal, i.e. $Y_{S}=\mathcal{E}_{S, \hat{\tau}}^{f}\left(\xi_{\hat{\tau}}\right)$ a.s. Since by Theorem 4.2 and by Proposition A.5, $Y$ is a strong $\mathcal{E}^{f}$-supermartingale, we have

$$
Y_{S} \geq \mathcal{E}_{S, \hat{\tau}}^{f}\left(Y_{\hat{\tau}}\right) \geq \mathcal{E}_{S, \hat{\tau}}^{f}\left(\xi_{\hat{\tau}}\right)=Y_{S} \quad \text { a.s. },
$$

where the last inequality holds because $Y \geq \xi$. It follows that $Y_{S}=\mathcal{E}_{S, \hat{\tau}}^{f}\left(Y_{\hat{\tau}}\right)$ a.s. Since $\theta_{t}^{y, z, k_{1}, k_{2}}>-1$, Property 4.1 can be applied, which yields that $Y$ is a strong $\mathcal{E}^{f}$-martingale on $[S, \hat{\tau}]$. Moreover, since $\mathcal{E}_{S, \hat{\tau}}^{f}\left(Y_{\hat{\tau}}\right)=\mathcal{E}_{S, \hat{\tau}}^{f}\left(\xi_{\hat{\tau}}\right)$ a.s. with $Y_{\hat{\tau}} \geq \xi_{\hat{\tau}}$ a.s., the strict monotonicity of $\mathcal{E}^{f}$ implies that $Y_{\hat{\tau}}=\xi_{\hat{\tau}}$ a.s.

We note that, even in the case where $\xi$ is right-continuous, the large inequality $\theta_{t}^{y, z, k_{1}, k_{2}} \geq$ -1 from Assumption 4.1 is not sufficient for the last statement of the above proposition to hold true; a counter-example is given in the Appendix (cf. Example A.2).

In Theorem 4.2 (ii), we have shown the existence of an $L \varepsilon$-optimal stopping time for problem (4.26). Under an additional assumption of left upper-semicontinuity along stopping times of the process $\xi$, we will show the existence of an optimal stopping time. To this purpose, we first give a lemma which is to be compared with Lemma 4.1.

Lemma 4.2 Let $f$ be a predictable Lipschitz driver satisfying Assumption 4.1. Let $\left(\xi_{t}, 0 \leq\right.$ $t \leq T)$ be a left-limited r.u.s.c. process in $\mathcal{S}^{2}$ which we assume also to be l.u.s.c. along stopping times. Let $(Y, Z, k, A, C)$ be the solution to the reflected BSDE with parameters $(f, \xi)$. Let $S \in \mathcal{T}_{0, T}$. We define $\tau_{S}^{*}$ by

$$
\tau_{S}^{*}:=\inf \left\{u \geq S: Y_{u}=\xi_{u}\right\} .
$$

The following assertions hold:

(i) $Y_{\tau_{S}^{*}}=\xi_{\tau_{S}^{*}}$ a.s.

(ii) The process $Y$ is a strong $\mathcal{E}^{f}$-martingale on $\left[S, \tau_{S}^{*}\right]$.

Proof: To prove the first statement we note that $Y_{\tau_{S}^{*}} \geq \xi_{\tau_{S}^{*}}$ a.s., since $Y$ is (the first component of) the solution to the RBSDE with barrier $\xi$. We show that $Y_{\tau_{S}^{*}} \leq \xi_{\tau_{S}^{*}}$ a.s. by using the assumption of right-upper semicontinuity on the process $\xi$; the arguments are similar to those used in the proof of part $(i)$ of Lemma 4.1 and are left to the reader.

Let us prove the second statement. By definition of $\tau_{S}^{*}$, we have that for a.e. $\omega \in \Omega, Y_{t}(\omega)>$ $\xi_{t}(\omega)$ on $\left[S(\omega), \tau_{S}^{*}(\omega)\left[\right.\right.$; hence, for a.e. $\omega$, the trajectory $A^{c}(\omega)$ is constant on $\left[S(\omega), \tau_{S}^{*}(\omega)[\right.$ and even on the closed interval $\left[S(\omega), \tau_{S}^{*}(\omega)\right]$ due to the continuity. On the other hand, due to the assumption of l.u.s.c. along stopping times on the process $\xi$, we have $A(\omega)=A^{c}(\omega)$ 
for a.e. $\omega$ (see Theorem 3.4). Thus, for a.e. $\omega, A(\omega)$ is constant on $\left[S(\omega), \tau_{S}^{*}(\omega)\right]$. We show that $C_{t-}(\omega)$ is constant on $\left[S(\omega), \tau_{S}^{*}(\omega)\right]$ by the same arguments as those of the proof of part (ii) of Lemma 4.1. We conclude by using Remark 4.8 .

Remark 4.10 We see from the above proof that the assumption of l.u.s.c. of $\xi$ in Lemma 4.2 can be replaced by the assumption $\Delta A_{\tau_{S}^{*}}=0$. The assumption $\Delta A_{\tau_{S}^{*}}=0$ is weaker than the assumption of l.u.s.c. of $\xi$ as illustrated in Example A.3 of the Appendix.

By the previous lemma and the first statement ("the optimality criterion") from Proposition 4.2 , we derive the following existence result.

Proposition 4.3 Let $f$ be a predictable Lipschitz driver satisfying Assumption 4.1. Let $\left(\xi_{t}, 0 \leq t \leq T\right)$ be a left-limited r.u.s.c. process in $\mathcal{S}^{2}$ which we assume also to be l.u.s.c. along stopping times. Let $S \in \mathcal{T}_{0, T}$. The stopping time $\tau_{S}^{*}$ defined in (4.36) is optimal for problem (4.32), that is $Y_{S}=\operatorname{ess}_{\sup } \operatorname{si}_{S, T} \mathcal{E}_{S, \tau}^{f}\left(\xi_{\tau}\right)=\mathcal{E}_{S, \tau_{S}^{*}}^{f}\left(\xi_{\tau_{S}^{*}}\right)$ a.s.

Remark 4.11 We note that, due to Remark 4.10 and to the optimality criterion, the optimality of $\tau_{S}^{*}$ in the above proposition still holds if we relax the assumption of l.u.s.c. of $\xi$ to the (weaker) assumption $\Delta A_{\tau_{S}^{*}}=0$ a.s. We recall that, by Remark 3.4, the condition $\Delta A_{\tau_{S}^{*}}=0$ a.s. is equivalent to $Y$ being left-continuous along stopping times at $\tau_{S}^{*}$. If the condition $\Delta A_{\tau_{S}^{*}}=0$ a.s. is violated, the stopping time $\tau_{S}^{*}$ might not be optimal (cf. Example A.3 from the Appendix).

We show the following property.

Proposition 4.4 Let $T>0$ be the terminal time. Let $\left(\xi_{t}, 0 \leq t \leq T\right)$ be a left-limited r.u.s.c. process in $\mathcal{S}^{2}$ and let $f$ be a predictable Lipschitz driver satisfying Assumption 4.1. Let $(Y, Z, k, A, C)$ be the solution to the reflected $B S D E$ with parameters $(\xi, f)$ as in Definition 3.1. The process $Y$ is the $\mathcal{E}^{f}$-Snell envelope of $\xi$, that is, the smallest strong $\mathcal{E}^{f}$-supermartingale greater than or equal to $\xi$.

Remark 4.12 This result still holds when $\xi$ is not left-limited (cf. Remarks 3.3 and 4.9).

From Proposition 4.4 and Theorem 4.2, we deduce that the "value process" of the optimal stopping problem (4.28) is characterized as the $\mathcal{E}^{f}$-Snell envelope of the reward process $\xi$. In the particular case of a classical (linear) expectation (that is, when $f=0$ ), we recover a characterization from the classical optimal stopping theory stating that the "value process" of the "classical" linear optimal stopping problem coincides with the Snell envelope of $\xi$, which is smallest strong supermartingale greater than or equal to $\xi$ (cf, e.g., [1]).

Proof: By Proposition A.5 in the Appendix, the process $Y$ is a strong $\mathcal{E}^{f}$-supermartingale. Moreover, since $Y$ is (the first component of) the solution to the reflected BSDE with 
parameters $(f, \xi)$, it is greater than or equal to $\xi$ (cf. Def. 3.1).

It remains to show the minimality property. Let $Y^{\prime}$ be another $\mathcal{E}^{f}$-supermartingale greater than or equal to $\xi$. Let $S \in \mathcal{T}_{0, T}$. For each $\tau \in \mathcal{T}_{S, T}$, we have $Y_{S}^{\prime} \geq \mathcal{E}_{S, \tau}^{f}\left(Y_{\tau}^{\prime}\right) \geq \mathcal{E}_{S, \tau}^{f}\left(\xi_{\tau}\right)$ a.s., where the second inequality follows from the inequality $Y^{\prime} \geq \xi$ and the monotonicity property of $\mathcal{E}^{f}$ with respect to the terminal condition. By taking the supremum over $\tau \in$ $\mathcal{T}_{S, T}$, we get $Y_{S}^{\prime} \geq \operatorname{ess}_{\sup _{\tau \in \mathcal{T}_{S, T}}} \mathcal{E}_{S, \tau}^{f}\left(\xi_{\tau}\right)=Y_{S}$ a.s., where the last equality follows from Theorem 4.2. The desired result follows.

\section{Additional results.}

5.1. $\mathcal{E}^{f}$-Mertens decomposition of $\mathcal{E}^{f}$-strong supermartingales. We now show an $\mathcal{E}^{f}$ Mertens decomposition for $\mathcal{E}^{f}$-strong supermartingales, which generalizes Mertens decomposition to the case of $f$-expectations. We first show the following lemma.

Lemma 5.1 Let $\left(Y_{t}\right) \in \mathcal{S}^{2}$ be a strong $\mathcal{E}^{f}$-supermartingale (resp. $\mathcal{E}^{f}$-submartingale). Then, $\left(Y_{t}\right)$ is right upper-semicontinuous (resp. right lower-semicontinuous).

Proof: Suppose that $\left(Y_{t}\right)$ is a strong $\mathcal{E}^{f}$-supermartingale. Let $\tau \in \mathcal{T}_{0, T}$ and let $\left(\tau_{n}\right)$ be a nonincreasing sequence of stopping times with $\lim _{n \rightarrow+\infty} \tau_{n}=\tau$ a.s. and for all $n \in \mathbb{N}$, $\tau_{n}>\tau$ a.s. on $\{\tau<T\}$. Suppose that $\lim _{n \rightarrow+\infty} Y_{\tau_{n}}$ exists a.s. The random variable $\lim _{n \rightarrow+\infty} Y_{\tau_{n}}$ is $\mathcal{F}_{\tau}$-measurable as the filtration is right-continuous. Let us show that

$$
Y_{\tau} \geq \lim _{n \rightarrow+\infty} Y_{\tau_{n}} \text { a.s. }
$$

Since $\left(Y_{t}\right)$ is a strong $\mathcal{E}^{f}$-supermartingale and the sequence $\left(\tau_{n}\right)$ is nonincreasing, we have, for all $n \in \mathbb{N}, \mathcal{E}_{\tau, \tau_{n}}^{f}\left(Y_{\tau_{n}}\right) \leq \mathcal{E}_{\tau, \tau_{n+1}}^{f}\left(Y_{\tau_{n+1}}\right) \leq Y_{\tau}$. We deduce that the sequence of random variables $\left(\mathcal{E}_{\tau, \tau_{n}}^{f}\left(Y_{\tau_{n}}\right)\right)_{n \in \mathbb{N}}$ is nondecreasing (hence, converges a.s.) and its limit (in the a.s. sense) satisfies $Y_{\tau} \geq \lim _{n \rightarrow+\infty} \uparrow \mathcal{E}_{\tau, \tau_{n}}^{f}\left(Y_{\tau_{n}}\right)$ a.s. This observation, combined with the continuity property of BSDEs with respect to terminal time and terminal condition (cf. [33, Prop. A.6]) gives

$$
Y_{\tau} \geq \lim _{n \rightarrow+\infty} \mathcal{E}_{\tau, \tau_{n}}^{f}\left(Y_{\tau_{n}}\right)=\mathcal{E}_{\tau, \tau}^{f}\left(\lim _{n \rightarrow+\infty} Y_{\tau_{n}}\right)=\lim _{n \rightarrow+\infty} Y_{\tau_{n}} \quad \text { a.s. }
$$

This result, together with a result of the general theory of processes (cf. [7, Prop. 2, page $300]$ ), ensures that the optional process $\left(Y_{t}\right)$ is right-upper semicontinuous.

Theorem $5.2\left(\mathcal{E}^{f}\right.$-Mertens decomposition) Let $\left(Y_{t}\right)$ be a process in $\mathcal{S}^{2}$. Let $f$ be a predictable Lipschitz driver satisfying Assumption 4.1. The process $\left(Y_{t}\right)$ is a strong $\mathcal{E}^{f}$ supermartingale (resp. $\mathcal{E}^{f}$-submartingale) if and only if there exists a nondecreasing (resp. 
nonincreasing) right-continuous predictable process $A$ in $\mathcal{S}^{2}$ with $A_{0}=0$ and a nondecreasing (resp. nonincreasing) right-continuous adapted purely discontinuous process $C$ in $\mathcal{S}^{2}$ with $C_{0-}=0$, as well as two processes $Z \in \mathbb{H}^{2}$ and $k \in \mathbb{H}_{\nu}^{2}$, such that a.s. for all $t \in[0, T]$, $Y_{t}=Y_{T}+\int_{t}^{T} f\left(s, Y_{s}, Z_{s}, k_{s}\right) d s+A_{T}-A_{t}+C_{T-}-C_{t-}-\int_{t}^{T} Z_{s} d W_{s}-\int_{t}^{T} \int_{E} k_{s}(e) \tilde{N}(d s, d e)$.

This decomposition is unique.

Proof: The "if part" has been shown in Proposition A.5 of the Appendix. Let us show the "only if" part. Suppose that $\left(Y_{t}\right)$ is a strong $\mathcal{E}^{f}$-supermartingale. Hence, $\left(Y_{t}\right)$ is clearly the $\mathcal{E}^{f}$-Snell envelope of $\left(Y_{t}\right)$, that is the smallest strong $\mathcal{E}^{f}$-supermartingale greater or equal to $\left(Y_{t}\right)$. By the characterization of the solution of a reflected BSDE as the $\mathcal{E}^{f}$-Snell envelope of the obstacle process (cf. Proposition 4.4 and Remark 4.12), we derive that the process $\left(Y_{t}\right)$ coincides with the solution of the reflected BSDE associated with the obstacle $\left(Y_{t}\right)$ (which is r.u.s.c. by Lemma 5.1). The desired conclusion follows.

The uniqueness of the processes $Z, k, A, C$ of the decomposition follows from the uniqueness of the solution of the reflected BSDE.

When $Y$ is right-continuous, the process $C$ of the $\mathcal{E}^{f}$-Mertens decomposition is equal to 0 . In this case, the previous theorem reduces to the so-called $\mathcal{E}^{f}$-Doob-Meyer decomposition (cf. Proposition A.6 in [10]; cf. also [36] and [31]).

Through different techniques, a similar result to the above Theorem 5.2 has been established in the recent paper [5] (in the Brownian framework).

Remark 5.13 It follows from the previous theorem that strong $\mathcal{E}^{f}$-supermartingales and strong $\mathcal{E}^{f}$-submartingales have left and right limits.

5.2. Comparison theorem for RBSDEs .

Theorem 5.3 (Comparison) Let $\xi^{1}$, $\xi^{2}$ be two obstacles. Let $f^{1}$ and $f^{2}$ be predictable Lipschitz drivers satisfying Assumption 4.1. Let $\left(Y^{i}, Z^{i}, k^{i}, A^{i}, C^{i}\right)$ be the solution of the $R B S D E$ associated with $\left(\xi^{i}, f^{i}\right), i=1,2$. Suppose that $\xi_{t}^{2} \leq \xi_{t}^{1}, 0 \leq t \leq T$ a.s. and that $f^{2}\left(t, Y_{t}^{2}, Z_{t}^{2}, k_{t}^{2}\right) \leq f^{1}\left(t, Y_{t}^{2}, Z_{t}^{2}, k_{t}^{2}\right), 0 \leq t \leq T d P \otimes d t$-a.s.

Then, $Y_{t}^{2} \leq Y_{t}^{1}, \forall t \in[0, T]$ a.s.

Proof: Step 1: Let us first consider the case where, along with the assumptions of the theorem, the following additional assumption holds: $f^{2}(t, y, z, k) \leq f^{1}(t, y, z, k)$ for all 
$(y, z, k) \in \mathbf{R}^{2} \times L_{\nu}^{2} d P \otimes d t$-a.s. Let $S \in \mathcal{T}_{0, T}$. By the comparison theorem for BSDEs, for each $\tau$ in $\mathcal{T}_{S, T}$ we have $\mathcal{E}_{S, \tau}^{f^{2}}\left(\xi_{\tau}^{2}\right) \leq \mathcal{E}_{S, \tau}^{f^{1}}\left(\xi_{\tau}^{1}\right)$ a.s. By taking the essential supremum over $\tau \in \mathcal{T}_{S, T}$ and by using Theorem 4.2 , we get $Y_{S}^{2} \leq Y_{S}^{1}$ a.s.

Step 2: Let us now place ourselves under the assumptions of the theorem (without the additional assumption on $f^{1}$ and $f^{2}$ from Step 1). Let $\delta f$ be the process defined by $\delta f_{t}:=f^{2}\left(t, Y_{t}^{2}, Z_{t}^{2}, k_{t}^{2}\right)-f^{1}\left(t, Y_{t}^{2}, Z_{t}^{2}, k_{t}^{2}\right)$. Note that $\left(Y^{2}, Z^{2}, k^{2}\right)$ is the solution of the reflected BSDE associated with obstacle $\xi^{2}$ and driver $f^{1}(t, y, z, k)+\delta f_{t}$. Now, by assumption, we have $f^{1}(t, y, z, k)+\delta f_{t} \leq f^{1}(t, y, z, k)$ for all $(y, z, k)$. By Step 1 applied to the drivers $f^{1}$ and $f^{1}(t, y, z, k)+\delta f_{t}\left(\right.$ instead of $\left.f^{2}\right)$, we get $Y^{2} \leq Y^{1}$.

6. Further developments. In our ongoing work (cf. [17]), we study the case of doubly reflected BSDEs where the barriers are not right-continuous.

\section{APPENDIX A}

The following observation is given for the convenience of the reader.

Remark A.14 Let $Y$ be a right-continuous (or left-continuous) adapted process. Then, $\sup _{t \in[0, T]} Y_{t}=\sup _{t \in[0, T] \cap \mathbb{Q}} Y_{t}$ a.s., which implies that $\sup _{t \in[0, T]} Y_{t}$ is a random variable.

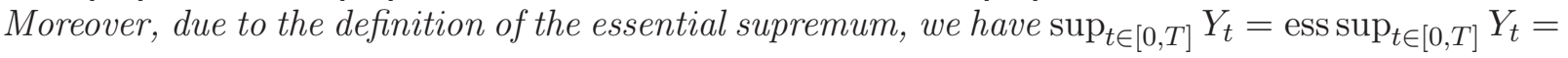
$\operatorname{ess}_{\sup } \operatorname{T}_{0, T} Y_{\tau}$ a.s.

Definition A.1 Let $(Y)_{t \in[0, T]}$ be an optional process. We say that $Y$ is a strong (optional) supermartingale if $Y_{\tau}$ is integrable for all $\tau \in \mathcal{T}_{0, T}$ and $Y_{S} \geq E\left[Y_{\tau} \mid \mathcal{F}_{S}\right]$ a.s., for all $S, \tau \in \mathcal{T}_{0, T}$ such that $S \leq \tau$ a.s.

We recall a decomposition of strong optional supermartingales, known as Mertens decomposition (see e.g. [9, Theorem 20, page 429, combined with Remark 3(b), page 205] and [9, Appendix 1, Thm.20, equalities (20.2)]).

Theorem A.1 (Mertens decomposition) Let $Y$ be a strong optional supermartingale of class $(D)$. There exists a unique right-continuous left-limited uniformly integrable martingale $\left(M_{t}\right)$, a unique predictable right-continuous nondecreasing process $\left(A_{t}\right)$ with $A_{0}=0$ and $E\left[A_{T}\right]<\infty$, and a unique right-continuous adapted nondecreasing process $\left(C_{t}\right)$, which is purely discontinuous, with $C_{0-}=0$ and $E\left[C_{T}\right]<\infty$, such that

$$
Y_{t}=M_{t}-A_{t}-C_{t-}, \quad 0 \leq t \leq T \text { a.s. }
$$

In particular, all trajectories of $Y$ have left and right limits.

Remark A.15 Since the filtration in our framework is quasi-left-continuous, martingales have only totally inaccessible jumps. From this and from Mertens decomposition (A.37), we deduce that, for each predictable stopping time $\tau, Y_{\tau}-Y_{\tau-}=-\left(A_{\tau}-A_{\tau-}\right)$ a.s. 
Remark A.16 By Mertens decomposition (A.37), we get $\Delta C_{t}=C_{t}-C_{t-}=Y_{t}-Y_{t+}$. Hence, $Y_{t} \geq Y_{t+}$, for all $t \in[0, T)$, which implies that $Y$ is necessarily r.u.s.c. Moreover, $Y$ is right-continuous if and only if $C \equiv 0$.

By using this remark, we recover the well-known Doob-Meyer decomposition for rightcontinuous supermartingales of class $(D)$. Indeed, let $Y$ be a right-continuous supermartingale (in the usual sense) of class $(D)$. Then $Y$ is a strong (optional) supermartingale in the sense of the above definition (due to the optional sampling theorem for right-continuous supermartingales). Mertens decomposition of $Y$ reduces to $Y=M-A$ (where $M$ and $A$ are as above), as $C \equiv 0$. This corresponds to Doob-Meyer decomposition of $Y$.

The following result from potential theory can be found in [9].

Theorem A.2 (Dellacherie-Meyer) Let $K$ be a non-decreasing predictable process (which is not necessarily right-continuous). Let $U$ be the potential of the process $K$, i.e.

$$
U_{t}:=E\left[K_{T}-K_{t} \mid F_{t}\right]
$$

for all $t \in[0, T]$. Assume that there exists a non-negative $\mathcal{F}_{T}$-measurable random variable $X$ such that $U_{S} \leq E\left[X \mid \mathcal{F}_{S}\right]$ a.s. for all $S \in \mathcal{T}_{0, T}$. Then, there exists a constant $c>0$ such that

$$
E\left[K_{T}^{2}\right] \leq c E\left[X^{2}\right]
$$

Proof: For the proof of the result the reader is referred to Paragraph 18 in [9, Appendix 1] generalizing Theorem VI.99 of the same reference to the case of a non-decreasing process which is not necessarily right-continuous nor left-continuous.

By using the previous theorem, we obtain the following integrability property of the Mertens process associated with a strong optional supermartingale, which is used in the proof of Lemma 3.3.

Corollary A.1 (Mertens process of a strong supermartingale: a useful estimate) Let $Y$ be a strong optional supermartingale of class $(D)$ such that: for all $S \in \mathcal{T}_{0, T}$, $\left|Y_{S}\right| \leq E\left[X \mid \mathcal{F}_{S}\right]$ a.s., where $X$ is a non-negative $\mathcal{F}_{T}$-measurable random variable.

Let us consider the Mertens process of $Y$, that is the process $\left(A_{t}+C_{t-}\right)$, where $A$ and $C$ are the two nondecreasing processes of Mertens decomposition of $Y$ from equation (A.37). There exists a constant $c>0$ such that

$$
E\left[\left(A_{T}+C_{T-}\right)^{2}\right] \leq c E\left[X^{2}\right]
$$


Proof: Let us introduce the notation $K_{t}:=A_{t}+C_{t-}(K$ is the Mertens process of $Y)$. Note that $K$ is a non-decreasing predictable process (which is not necessarily right-continuous). Let $S \in \mathcal{T}_{0, T}$. From Mertens decomposition, we have $Y_{S}=M_{S}-K_{S}$ a.s. and $Y_{T}=$ $M_{T}-K_{T}$ a.s. By subtracting the second equation from the first, and by taking conditional expectations, we derive that $Y_{S}-E\left[Y_{T} \mid \mathcal{F}_{S}\right]=E\left[K_{T}-K_{S} \mid \mathcal{F}_{S}\right]$ a.s. Hence, the process $\left(U_{t}\right)$ defined by $U_{t}:=Y_{t}-E\left[Y_{T} \mid \mathcal{F}_{t}\right]$ is the potential associated with the non-decreasing predictable process $K$. Now, we have

$$
\left|U_{S}\right|=\left|Y_{S}-E\left[Y_{T} \mid \mathcal{F}_{S}\right]\right| \leq\left|Y_{S}\right|+E\left[\left|Y_{T}\right| \mid \mathcal{F}_{S}\right] \leq E\left[2 X \mid \mathcal{F}_{S}\right] \text { a.s. },
$$

where the last inequality follows from the assumption. By applying Theorem A.2, there exists a constant $c>0$ such that $E\left[K_{T}^{2}\right] \leq c E\left[X^{2}\right]$, which is the desired conclusion.

We recall the change of variables formula for optional strong semimartingales which are not necessarily right-continuous. The result can be seen as a generalization of the classical Itô formula and can be found in [16, Theorem 8.2] (cf. also [26, Chapter VI, Section 3, page $538])$. We recall the result in our framework in which the underlying filtered probability space satisfies the usual conditions.

Theorem A.3 (Gal'chouk-Lenglart) Let $n \in \mathbb{N}$. Let $X$ be an $n$-dimensional optional strong semimartingale, i.e. $X=\left(X^{1}, \ldots, X^{n}\right)$ is an $n$-dimensional optional process with decomposition $X^{k}=X_{0}^{k}+M^{k}+A^{k}+B^{k}$, for all $k \in\{1, \ldots, n\}$, where $M^{k}$ is a (cadlag) local martingale, $A^{k}$ is a right-continuous adapted process of finite variation such that $A_{0}=0$, and $B^{k}$ is a left-continuous adapted process of finite variation which is purely discontinuous and such that $B_{0}=0$. Let $F$ be a twice continuously differentiable function on $\mathbf{R}^{n}$. Then,

$$
\begin{aligned}
F\left(X_{t}\right)= & F\left(X_{0}\right)+\sum_{k=1}^{n} \int_{] 0, t]} D^{k} F\left(X_{s-}\right) d\left(A^{k}+M^{k}\right)_{s}+\frac{1}{2} \sum_{k, l=1}^{n} \int_{] 0, t]} D^{k} D^{l} F\left(X_{s-}\right) d<M^{k c}, M^{l c}>_{s} \\
& +\sum_{0<s \leq t}\left[F\left(X_{s}\right)-F\left(X_{s-}\right)-\sum_{k=1}^{n} D^{k} F\left(X_{s-}\right) \Delta X_{s}^{k}\right]+\sum_{k=1}^{n} \int_{[0, t[} D^{k} F\left(X_{s}\right) d\left(B^{k}\right)_{s+} \\
& +\sum_{0 \leq s<t}\left[F\left(X_{s+}\right)-F\left(X_{s}\right)-\sum_{k=1}^{n} D^{k} F\left(X_{s}\right) \Delta_{+} X_{s}^{k}\right], \quad 0 \leq t \leq T \quad \text { a.s. },
\end{aligned}
$$

where $D^{k}$ denotes the differentiation operator with respect to the $k$-th coordinate, and $M^{k c}$ denotes the continuous part of $M^{k}$.

Corollary A.2 Let $Y$ be a one-dimensional optional strong semimartingale with decomposition $Y=Y_{0}+M+A+B$, where $M, A$, and $B$ are as in the above theorem. Let $\beta>0$. 
Then, almost surely, for all $t \geq 0$,

$$
\begin{aligned}
\mathrm{e}^{\beta t} Y_{t}^{2}=Y_{0}^{2} & +\int_{] 0, t]} \beta \mathrm{e}^{\beta s} Y_{s}^{2} d s+2 \int_{] 0, t]} \mathrm{e}^{\beta s} Y_{s-} d(A+M)_{s}+\int_{] 0, t]} \mathrm{e}^{\beta s} d<M^{c}, M^{c}>_{s} \\
& +\sum_{0<s \leq t} \mathrm{e}^{\beta s}\left(Y_{s}-Y_{s-}\right)^{2}+\int_{[0, t[} 2 \mathrm{e}^{\beta s} Y_{s} d(B)_{s+}+\sum_{0 \leq s<t} \mathrm{e}^{\beta s}\left(Y_{s+}-Y_{s}\right)^{2} .
\end{aligned}
$$

Proof: It suffices to apply Gal'chouk-Lenglart's formula with $n=2, F(x, y)=x y^{2}, X_{t}^{1}=$ $\mathrm{e}^{\beta t}$, and $X_{t}^{2}=Y_{t}$. Indeed, by applying Theorem A.3 and by noting that the local martingale part and the purely discontinuous part of $X^{1}$ are both equal to 0 , we obtain

$$
\begin{aligned}
\mathrm{e}^{\beta t} Y_{t}^{2} & =Y_{0}^{2}+\int_{] 0, t]} \beta \mathrm{e}^{\beta s} Y_{s}^{2} d s+2 \int_{] 0, t]} \mathrm{e}^{\beta s} Y_{s-} d(A+M)_{s}+\frac{1}{2} \int_{] 0, t]} 2 \mathrm{e}^{\beta s} d<M^{c}, M^{c}>_{s} \\
& +\sum_{0<s \leq t} \mathrm{e}^{\beta s}\left(Y_{s}^{2}-\left(Y_{s-}\right)^{2}-2 Y_{s-}\left(Y_{s}-Y_{s-}\right)\right) \\
& +\int_{[0, t[} 2 \mathrm{e}^{\beta s} Y_{s} d(B)_{s+}+\sum_{0 \leq s<t} \mathrm{e}^{\beta s}\left(\left(Y_{s+}\right)^{2}-\left(Y_{s}\right)^{2}-2 Y_{s}\left(Y_{s+}-Y_{s}\right)\right) .
\end{aligned}
$$

The desired expression follows as $Y_{s}^{2}-\left(Y_{s-}\right)^{2}-2 Y_{s-}\left(Y_{s}-Y_{s-}\right)=\left(Y_{s}-Y_{s-}\right)^{2}$ and $\left(Y_{s+}\right)^{2}-$ $\left(Y_{s}\right)^{2}-2 Y_{s}\left(Y_{s+}-Y_{s}\right)=\left(Y_{s+}-Y_{s}\right)^{2}$.

Proposition A.5 (BSDE with "generalized" driver vs. BSDE) Let $f$ be a predictable Lipschitz driver satisfying Assumption 4.1. Let $A$ be a nondecreasing (resp. nonincreasing) right-continuous predictable process in $\mathcal{S}^{2}$ with $A_{0}=0$ and let $C$ be a nondecreasing (resp. nonincreasing) right-continuous adapted purely discontinuous process in $\mathcal{S}^{2}$ with $C_{0-}=0$. Let $(Y, Z, k) \in \mathcal{S}^{2} \times \mathbb{H}^{2} \times \mathbb{H}_{\nu}^{2}$ satisfy a.s. for all $t \in[0, T]$,

$Y_{t}=Y_{T}+\int_{t}^{T} f\left(s, Y_{s}, Z_{s}, k_{s}\right) d s+A_{T}-A_{t}+C_{T-}-C_{t-}-\int_{t}^{T} Z_{s} d W_{s}-\int_{t}^{T} \int_{E} k_{s}(e) \tilde{N}(d s, d e)$.

Then the process $\left(Y_{t}\right)$ is a strong $\mathcal{E}^{f}$-supermartingale (resp. $\mathcal{E}^{f}$-submartingale).

Proof: We address the case where $A$ and $C$ are nondecreasing. Let $\tau, \theta \in \mathcal{T}_{0}$ be such that $\tau \leq \theta$ a.s. Let us show that $Y_{\tau} \geq \mathcal{E}_{\tau, \theta}^{f}\left(Y_{\theta}\right)$ a.s.

We denote by $(X, \pi, l)$ the solution to the BSDE associated with driver $f$, terminal time $\theta$, and terminal condition $Y_{\theta}$; then $\mathcal{E}_{\tau, \theta}^{f}\left(Y_{\theta}\right)=X_{\tau}$ a.s. (by definition of $\mathcal{E}^{f}$ ).

Set $\bar{Y}_{t}=Y_{t}-X_{t}, \bar{Z}_{t}=Z_{t}-\pi_{t}$ and $\bar{k}_{t}=k_{t}-l_{t}$. Then

$$
-d \bar{Y}_{t}=h_{t} d t+d A_{t}+d C_{t-}-\bar{Z}_{t} d W_{t}-\int_{E} \bar{k}_{t}(e) \tilde{N}(d t, d e), \quad \bar{Y}_{\theta}=0,
$$


where $h_{t}:=f\left(t, Y_{t-}, Z_{t}, k_{t}\right)-f\left(t, X_{t-}, \pi_{t}, l_{t}\right)$. By the same arguments as those of the proof of the comparison theorem for BSDEs with jumps (cf. [33, Thm. 4.2], or [36]), using Assumption 4.1 on $f$, we can show that

$$
h_{t} \geq \delta_{t} \bar{Y}_{t}+\beta_{t} \bar{Z}_{t}+\left\langle\gamma_{t}, \bar{k}_{t}\right\rangle_{\nu}, \quad 0 \leq t \leq T, \quad d P \otimes d t-\text { a.e. }
$$

where $\gamma_{t}:=\theta_{t}^{X_{t-}, \pi_{t}, k_{t}, l_{t}}$ and where $\delta$ and $\beta$ are predictable bounded processes (which can be expressed as increment rates of $f$ with respect to $y$ and $z$ ).

Let $\Gamma_{\tau}$. be the unique solution of the following forward SDE

$$
d \Gamma_{\tau, s}=\Gamma_{\tau, s-}\left[\delta_{s} d s+\beta_{s} d W_{s}+\int_{E} \gamma_{s}(e) \tilde{N}(d s, d e)\right] ; \quad \Gamma_{\tau, \tau}=1
$$

Suppose for a while that we have shown

$$
\Gamma_{\tau, \tau} \bar{Y}_{\tau} \geq E\left[\int_{\tau}^{\theta} \Gamma_{\tau, s-} d A_{s}+\int_{\tau}^{\theta} \Gamma_{\tau, s} d C_{s} \mid \mathcal{F}_{\tau}\right] \text { a.s. }
$$

Then, since $\Gamma_{\tau, s} \geq 0$ and $\Gamma_{\tau, \tau}=1$, we have $\bar{Y}_{\tau} \geq 0$ a.s., that is $Y_{\tau} \geq X_{\tau}=\mathcal{E}_{\tau, \theta}^{f}\left(Y_{\theta}\right)$ a.s. which is the desired result. It remains to show (A.44). To simplify the notation, we denote $\Gamma_{\tau, s}$ by $\Gamma_{s}$ for $s \geq \tau$. We use that $\bar{Y}$ is a strong optional semimartingale with decomposition $\bar{Y}=M^{1}+A^{1}+B^{1}$, where $M_{t}^{1}=\int_{0}^{t} \bar{Z}_{s} d W_{s}+\int_{0}^{t} \int_{E} \bar{k}_{s}(e) \tilde{N}(d s, d e), A_{t}^{1}:=-\int_{0}^{t} h_{s} d s-A_{s}$, and $B_{t}^{1}:=-C_{t-}$, and we apply Gal'chouk-Lenglart's formula from Theorem A.3 with $n:=2$, $X^{1}:=\bar{Y}, X^{2}:=\Gamma$, and $F\left(x^{1}, x^{2}\right):=x^{1} x^{2}$. We obtain

$$
\begin{aligned}
\Gamma_{\tau} \bar{Y}_{\tau} & =-\int_{\tau}^{\theta} \Gamma_{s-}\left(\bar{Z}_{s}+\bar{Y}_{s-} \beta_{s}\right) d W_{s}-\int_{\tau}^{\theta} \Gamma_{s}\left(\bar{Y}_{s-} \delta_{s}+\bar{Z}_{s} \beta_{s}-h_{s}\right) d s \\
& +\int_{\tau}^{\theta} \Gamma_{s-} d A_{s}+\int_{\tau}^{\theta} \Gamma_{s} d C_{s}-\int_{\tau}^{\theta} \int_{E} \Gamma_{s-}\left(\bar{k}_{s}(e)+\bar{Y}_{s-} \gamma_{s}(e)\right) \tilde{N}(d s, d e)-\sum_{\tau \leq s \leq \theta} \Delta \Gamma_{s} \Delta Y_{s} .
\end{aligned}
$$

By using the fact that $A$. and $N(\cdot$, de $)$ do not have common jumps, we get $\sum_{\tau \leq s \leq \theta} \Delta \Gamma_{s} \Delta Y_{s}=$ $\int_{\tau}^{\theta} \int_{E} \Gamma_{s-} \gamma_{s}(e) \bar{k}_{s}(e) N(d s, d e)$. By replacing this expression in equation (A.45) and by doing some computations, we obtain

$$
\begin{aligned}
\Gamma_{\tau} \bar{Y}_{\tau} & =-\int_{\tau}^{\theta} \Gamma_{s-}\left(\bar{Z}_{s}+\bar{Y}_{s-} \beta_{s}\right) d W_{s}-\int_{\tau}^{\theta} \Gamma_{s}\left(\bar{Y}_{s-} \delta_{s}+\bar{Z}_{s} \beta_{s}+\left\langle\gamma_{s}, \bar{k}_{s}\right\rangle_{\nu}-h_{s}\right) d s \\
& +\int_{\tau}^{\theta} \Gamma_{s-} d A_{s}+\int_{\tau}^{\theta} \Gamma_{s} d C_{s}-\int_{\tau}^{\theta} \int_{E} \Gamma_{s-}\left(\bar{k}_{s}(e)+\bar{Y}_{s-} \gamma_{s}(e)+\gamma_{s}(e) \bar{k}_{s}(e)\right) \tilde{N}(d s, d e) .
\end{aligned}
$$

Now, the stochastic integral with respect to " $d W_{s}$ " in the above equation is a martingale (since $\Gamma \in S^{2}, \bar{Z} \in \mathbb{H}^{2}, \bar{Y} \in \mathcal{S}^{2}$, and $\beta$ is bounded). The stochastic integral with respect 
to the Poisson random measure is also a martingale. By taking the conditional expectation and by using the inequality (A.42), we derive (A.44). The proof is thus complete.

Proof of Proposition 2.1: We first show that $\|\cdot\|_{\mathcal{S}^{2}}$ is a norm on the space of optional processes. The positive homogeneity and the triangular inequality are easy to check. Suppose

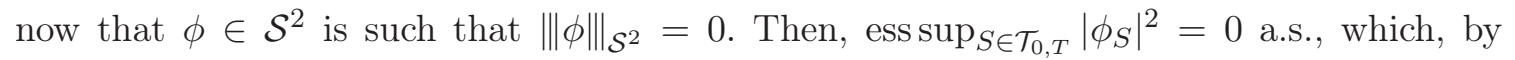
definition of the essential supremum, implies that $\left|\phi_{S}\right|^{2}=0$ a.s. for all $S \in \mathcal{T}_{0, T}$. By a classical result of the General Theory of Processes ([8, Theorem IV.84]), we obtain that the process $\phi$ is indistinguishable from the null process, that is $\phi_{t}=0,0 \leq t \leq T$ a.s. We conclude that $\|\cdot \cdot\|_{\mathcal{S}^{2}}$ is a norm on $\mathcal{S}^{2}$.

Let us prove that the space $\left(\mathcal{S}^{2},\|\cdot\|_{\mathcal{S}^{2}}\right)$ is complete. We only sketch the proof since its main steps are similar to those of the proof of the completeness of the space $\left(L^{2},\|\cdot\|_{L^{2}}\right)$. Let $\left(\phi^{n}\right)$ be a Cauchy sequence in $\mathcal{S}^{2}$ for the norm $\|\cdot\|_{\mathcal{S}^{2}}$. We extract a subsequence $\left(\phi^{n_{k}}\right)_{k \in \mathbb{N}}$ such that $\left\|\phi^{n_{k+1}}-\phi^{n_{k}}\right\|_{\mathcal{S}^{2}} \leq \frac{1}{2^{k}}$, for all $k \in \mathbb{N}$. Setting $g_{t}^{n}:=\sum_{k=1}^{n}\left|\phi_{t}^{n_{k+1}}-\phi^{n_{k}}\right|$ for each $n$, by the triangular inequality, we derive that $\left\|g^{n}\right\|_{\mathcal{S}^{2}} \leq \sum_{k=1}^{n}\|\| \phi_{t}^{n_{k+1}}-\phi^{n_{k}}\|\|_{\mathcal{S}^{2}} \leq$ $\sum_{k=1}^{n} \frac{1}{2^{k}} \leq \sum_{k=1}^{\infty} \frac{1}{2^{k}}=1$. We set $g_{t}:=\lim _{n} \uparrow g_{t}^{n}$, for all $t \in[0, T]$ (the limit exists in $[0,+\infty]$ as the sequence $\left(g_{t}^{n}\right)_{n}$ is non-negative non-decreasing). Being the limit of optional

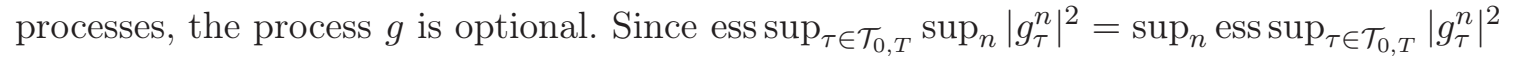
a.s., using the monotone convergence theorem, we derive that $\|g\|_{\mathcal{S}^{2}}=\lim _{n} \uparrow\|\|^{n} \|_{\mathcal{S}^{2}}$. As the sequence $\left(\left\|g^{n}\right\|_{\mathcal{S}^{2}}\right)$ is bounded by 1 , we get $\|g\|_{\mathcal{S}^{2}} \leq 1$. We then adapt the arguments from the proof of the completeness of $\left(L^{2},\|\cdot\|_{L^{2}}\right)$ to show that $\lim _{n}\left\|g-g^{n}\right\|_{\mathcal{S}^{2}}=0$, and that $\left\|\phi-\phi^{n_{l}}\right\|_{\mathcal{S}^{2}} \underset{l \rightarrow \infty}{\longrightarrow} 0$, which concludes the proof.

The following result of the optimal stopping theory is used in the proof of Lemma 3.3.

Proposition A.6 Let $(\bar{Y}(S))$ be the family defined for $S \in \mathcal{T}_{0, T}$ by

$$
\bar{Y}(S):=\operatorname{esssup}_{\tau \in \mathcal{T}_{S, T}} E\left[\xi_{\tau}+\int_{S}^{\tau} f(u) d u \mid \mathcal{F}_{S}\right]
$$

(i) There exists a ladlag optional process $\left(\bar{Y}_{t}\right)_{t \in[0, T]}$ which aggregates the family $(\bar{Y}(S))$ (i.e. $\bar{Y}_{S}=\bar{Y}(S)$, for all $\left.S \in \mathcal{T}_{0, T}\right)$.

Moreover, the process $\left(\bar{Y}_{t}+\int_{0}^{t} f(u) d u\right)_{t \in[0, T]}$ is a strong supermartingale.

(ii) We have $\bar{Y}_{S}=\xi_{S} \vee \bar{Y}_{S+}$ a.s. for all $S$.

(iii) Furthermore, $\bar{Y}_{S+}=\operatorname{ess}_{\sup _{\tau>S}} E\left[\xi_{\tau}+\int_{S}^{\tau} f(u) d u \mid \mathcal{F}_{S}\right]$ a.s., for all $S$.

Remark A.17 It follows from (ii) that $\Delta_{+} \bar{Y}_{S}=\mathbf{1}_{\left\{\bar{Y}_{S}=\xi_{S}\right\}} \Delta_{+} \bar{Y}_{S}$ a.s.

Proof: For completeness, we give here a short proof (cf. [27] when $\xi$ is left- and right-limited, 
and [22, Sect.B] in the general case). For $S \in \mathcal{T}_{0, T}$, we define $\overline{\bar{Y}}(S)$ by

$$
\overline{\bar{Y}}(S):=\bar{Y}(S)+\int_{0}^{S} f(u) d u=\operatorname{essip}_{\tau \in \mathcal{T}_{S, T}} E\left[\xi_{\tau}+\int_{0}^{\tau} f(u) d u \mid \mathcal{F}_{S}\right]
$$

where the equality follows from the definition of $\bar{Y}(S)$ (see (A.47)). For $S \in \mathcal{T}_{0, T}$, define

$$
\overline{\bar{Y}}^{+}(S):=\operatorname{esssup}_{\tau>S} E\left[\xi_{\tau}+\int_{0}^{\tau} f(u) d u \mid \mathcal{F}_{S}\right]
$$

By some well-known results of optimal stopping theory (cf., e.g. [21, Prop. D.3] or [22, Prop. 1.12]), the family of random variables $\left(\overline{\bar{Y}}^{+}(S)\right)$ is a supermartingale family which is right-continuous along stopping times in expectation. By classical results (cf., e.g., [11] or [25, Prop. 4.1]), there exists a process $\left(\overline{\bar{Y}}_{t}^{+}\right)$which aggregates this family. By [21, Prop. D.3] (cf. also [22, Prop. 1.9]), we have

$$
\overline{\bar{Y}}(S)=\left(\xi_{S}+\int_{0}^{S} f(u) d u\right) \vee \overline{\bar{Y}}^{+}(S) \text { a.s. },
$$

for all $S \in \mathcal{T}_{0, T}$. It follows that the process $\left(\overline{\bar{Y}}_{t}\right)$ defined by

$$
\overline{\bar{Y}}_{t}:=\left(\xi_{t}+\int_{0}^{t} f(u) d u\right) \vee \overline{\bar{Y}}_{t}^{+}
$$

aggregates the family $(\overline{\bar{Y}}(S))$. Since $(\overline{\bar{Y}}(S))$ is a supermartingale family, $\left(\overline{\bar{Y}}_{t}\right)$ is a strong supermartingale. Now, we know (cf., e.g., [22, Prop. 4.14], combined with [24, Appendix A1, paragraph 1]) that $\overline{\bar{Y}}^{+}(S)=\overline{\bar{Y}}(S+)$, for all $S \in \mathcal{T}_{0, T}$, where $\overline{\bar{Y}}(S+)$ denotes the right-hand limit of $\overline{\bar{Y}}$ along stopping times at $S$, as defined, for instance, in [22, Def. 4.5]. On the other hand, we know that the process $\left(\overline{\bar{Y}}_{t}\right)$ aggregates the family $(\overline{\bar{Y}}(S))$, which entails that the process $\left(\overline{\bar{Y}}_{t+}\right)$ aggregates the family $(\overline{\bar{Y}}(S+))$. By using Eq. (A.50), we conclude that

$$
\overline{\bar{Y}}_{S}=\left(\xi_{S}+\int_{0}^{S} f(u) d u\right) \vee \overline{\bar{Y}}_{S+} \text { a.s. }
$$

for all $S$. By (A.48), we derive $\bar{Y}(S)=\overline{\bar{Y}}(S)-\int_{0}^{S} f(u) d u=\overline{\bar{Y}}_{S}-\int_{0}^{S} f(u) d u$ a.s., for all $S \in \mathcal{T}_{0, T}$. The ladlag optional process $\left(\bar{Y}_{t}\right)_{t \in[0, T]}=\left(\overline{\bar{Y}}_{t}-\int_{0}^{t} f(u) d u\right)_{t \in[0, T]}$ thus aggregates the family $(\bar{Y}(S))_{S \in \mathcal{T}_{0, T}}$. Moreover, $\left(\bar{Y}_{t}+\int_{0}^{t} f(u) d u\right)=\left(\overline{\bar{Y}}_{t}\right)$ is a strong supermartingale, which gives (i). By using (A.52), we derive (ii). By using (A.49), we obtain (iii). 
Example A.1 (A toy example) Let $\left(\xi_{t}\right)$ be a deterministic continuous decreasing bounded function. We set $Y_{t}:=\sup _{s>t} \xi_{s}=\xi_{t}$ and $A_{t}:=\xi_{0}-\xi_{t}$, for all $t \in[0, T]$. It is wellknown (cf. [12], or the classical Skorokhod's problem as recalled in [21]) that $(Y, 0,0, A)$ is the unique solution to the RBSDE with driver $f \equiv 0$ and (continuous) obstacle $\xi$. Let us now change the obstacle $\xi$ at a single point $t_{0} \in[0, T)$. More precisely, we consider a function $\bar{\xi}$ such that $\bar{\xi}_{t}=\xi_{t}$, for $t \neq t_{0}$, and $\bar{\xi}_{t_{0}}>\xi_{t_{0}}$. We note that $\bar{\xi}$ is r.u.s.c. but not right-continuous. In this very simple example, we can compute explicitly a solution to the RBSDE (defined in Definition 3.1) with parameters $(0, \bar{\xi})$. We set $\bar{Y}_{t}:=\sup _{s \geq t} \bar{\xi}_{s}$, for $t \in[0, T]$. We first rewrite $\bar{Y}$ in a different manner. For $t>t_{0}$, we have $\bar{Y}_{t}=\xi_{t}=Y_{t}$. For $t \leq t_{0}$, we have $\bar{Y}_{t}=\max \left(\sup _{s \geq t, s \neq t_{0}} \xi_{s}, \bar{\xi}_{t_{0}}\right)=\max \left(\sup _{s \geq t} \xi_{s}, \bar{\xi}_{t_{0}}\right)=\max \left(\xi_{t}, \bar{\xi}_{t_{0}}\right)$. We set $t_{1}:=\sup \left\{s \geq 0: \xi_{s} \geq \bar{\xi}_{t_{0}}\right\}$, with the convention $\sup (\varnothing)=0$. We note that $\bar{Y}_{t}=\bar{\xi}_{t_{0}}$, for $t \in\left[t_{1}, t_{0}\right]$, and $\bar{Y}_{t}=\xi_{t}=Y_{t}$, for $t \in\left[0, t_{1}\right)$. We define $\bar{C}_{t}:=\left(\bar{\xi}_{t_{0}}-\xi_{t_{0}}\right) \mathbf{1}_{t \geq t_{0}}$, for $t \in[0, T]$. We see that $\bar{C}$ is non-decreasing, cad-lag, purely discontinuous (in fact, $\bar{C}$ has one single jump), and it satisfies the minimality condition (3.4). We now consider the following two cases: $(i)$ the case $\bar{\xi}_{t_{0}} \geq \xi_{t_{0}}$, and (ii) the case $\bar{\xi}_{t_{0}}<\xi_{t_{0}}$. In the case $(i)$, we have $t_{1}=0$; we set $\bar{A}_{t}:=0$, for $t \in\left[0, t_{0}\right]$, and $\bar{A}_{t}:=\xi_{t_{0}}-\xi_{t}$, for $t \in\left(t_{0}, T\right]$. In the case $(i i)$, we have $t_{1}>0$ and $\xi_{t_{1}}=\bar{\xi}_{t_{0}}$; we define $\bar{A}_{t}$ by $\bar{A}_{t}:=\xi_{0}-\xi_{t}$, for $t \in\left[0, t_{1}\right), \bar{A}_{t}:=\xi_{0}-\xi_{t_{1}}$, for $t \in\left[t_{1}, t_{0}\right]$, and $\bar{A}_{t}:=\xi_{0}-\xi_{t_{1}}+\xi_{t_{0}}-\xi_{t}$, for $t \in\left(t_{0}, T\right]$. In both cases, the function $\bar{A}$ is non-decreasing, continuous, and it satisfies the minimality condition (3.3). Moreover, it can be easily checked that, $\bar{Y}_{t}=\bar{\xi}_{T}+\bar{A}_{T}-\bar{A}_{t}+\bar{C}_{T-}-\bar{C}_{t-}$, for all $t \in[0, T]$. We conclude that $(\bar{Y}, 0,0, \bar{A}, \bar{C})$ is a solution to the reflected BSDE with parameters $(0, \bar{\xi})$. We prove in Lemma 3.3 that $(\bar{Y}, 0,0, \bar{A}, \bar{C})$ is the unique solution. We notice that $\bar{Y}$ has a jump on the right at $t_{0}$; the size $\Delta_{+} \bar{Y}_{t_{0}}$ of the jump satisfies $\Delta_{+} \bar{Y}_{t_{0}}:=\bar{Y}_{t_{0}+}-\bar{Y}_{t_{0}}=\xi_{t_{0}}-\bar{\xi}_{t_{0}}=-\left(C_{t_{0}}-C_{t_{0-}}\right)$.

Example A.2 (Counter-example) Let $\nu(d u):=\delta_{1}(d u)$, where $\delta_{1}$ denotes the Dirac measure at 1 . The process $N_{t}:=N([0, t] \times\{1\})$ is then a Poisson process with parameter 1 , and we have $\tilde{N}_{t}:=\tilde{N}([0, t] \times\{1\})=N_{t}-t$. Let the driver $f$ be given by $f(t, y, z, \ell):=$ $\langle-1, \ell\rangle_{\nu}=-\ell(1)$. We introduce the associated adjoint process $\Gamma_{t, .}$, defined for each $r \in[t, T]$ by $\Gamma_{t, r}=\mathbf{1}_{\left\{N_{r}-N_{t}=0\right\}} e^{r-t}$. Let the pay-off process $\xi$ be given by $\xi_{t}:=\mathbf{1}_{\left\{N_{t} \geq 1\right\}} e^{-t}$, for all $t \in$ $[0, T]$. Note that $\xi$ is adapted and right-continuous. By the representation property for linear BSDEs with jumps ([33, Thm.3.4]) and classical computations, we get

$$
\mathcal{E}_{t, \tau}^{f}\left(\xi_{\tau}\right)=E\left[\Gamma_{t, \tau} \xi_{\tau} \mid \mathcal{F}_{t}\right]=\mathrm{e}^{-t} E\left[\mathbf{1}_{\left\{N_{\tau}-N_{t}=0\right\}} \mathbf{1}_{\left\{N_{\tau} \geq 1\right\}} \mid \mathcal{F}_{t}\right]=\mathrm{e}^{-t} \mathbf{1}_{\left\{N_{t} \geq 1\right\}} E\left[\mathbf{1}_{\left\{N_{\tau}-N_{t}=0\right\}} \mid \mathcal{F}_{t}\right],
$$

for all $t \in[0, T]$, for all $\tau \in \mathcal{T}_{t, T}$. We deduce that $Y_{t}:=\operatorname{ess}_{\sup } \operatorname{si}_{t, T} \mathcal{E}_{t, \tau}^{f}\left(\xi_{\tau}\right)=\mathrm{e}^{-t} \mathbf{1}_{\left\{N_{t} \geq 1\right\}}=$ $\xi_{t}$, for all $t \in[0, T]$ (as $E\left[\mathbf{1}_{\left\{N_{\tau}-N_{t}=0\right\}} \mid \mathcal{F}_{t}\right] \leq 1$ and the upper bound is attained for $\tau=t$ ). Let us focus on the optimal stopping problem at time $t=0$. The above computations imply that, for $t=0, Y_{0}:=\operatorname{ess}_{\sup _{\tau} \in \mathcal{T}_{0, T}} \mathcal{E}_{0, \tau}^{f}\left(\xi_{\tau}\right)=\xi_{0}=0$. Moreover, the essential supremum (at time 0 ) is attained at any stopping time $\tau \in \mathcal{T}_{0, T}$ (indeed, $\mathcal{E}_{0, \tau}^{f}\left(\xi_{\tau}\right)=0$, for all $\tau \in \mathcal{T}_{0, T}$ ). 
This is true, in particular, for the stopping time $\hat{\tau}$ defined by $\hat{\tau}:=T$. However, we will see that the process $Y$ (computed above) is not an $\mathcal{E}^{f}$-martingale on $[0, \hat{\tau}]$. To do so, let us denote by $X$ the (first component) of the solution to the BSDE with driver $f$, terminal time $T$, and terminal condition $Y_{T}=\xi_{T}$. For $u \in[0, \hat{\tau}]=[0, T]$, we have $X_{u}=\mathcal{E}_{u, T}^{f}\left(\xi_{T}\right)=$ $\mathrm{e}^{-u} \mathbf{1}_{\left\{N_{u} \geq 1\right\}} E\left[\mathbf{1}_{\left\{N_{T}-N_{u}=0\right\}} \mid \mathcal{F}_{u}\right]=\mathrm{e}^{-u} \mathbf{1}_{\left\{N_{u} \geq 1\right\}} P\left[N_{T}-N_{u}=0\right]=\mathrm{e}^{-T} \mathbf{1}_{\left\{N_{u} \geq 1\right\}}$. Hence, for $u \in(0, T)$, we have $Y_{u}=\mathrm{e}^{-u}>\mathrm{e}^{-T}=X_{u}$ on the set $\left\{N_{u} \geq 1\right\}$. Hence, the processes $X$ and $Y$ are not indistinguishable.

Let us also note that in this example $\mathcal{E}^{f}$ is not strictly monotonous. To see this, we consider $\xi^{1}:=0$ and $\xi^{2}:=\xi_{T}=\mathrm{e}^{-T} \mathbf{1}_{\left\{N_{T} \geq 1\right\}}$. We have $\xi^{1} \leq \xi^{2}$ and $\mathcal{E}_{0, T}^{f}\left(\xi^{1}\right)=\mathcal{E}_{0, T}^{f}(0)=0=$ $\mathcal{E}_{0, T}^{f}\left(\xi_{T}\right)=\mathcal{E}_{0, T}^{f}\left(\xi^{2}\right)$. However, $\xi_{T} \neq 0$ with a positive probability.

Example A.3 Let $\left(\xi_{t}\right)$ be an RCLL deterministic bounded function, increasing on $\left[0, t_{0}[\right.$, decreasing on $\left[t_{0}, T\right]$, and supposed to be continuous on $[0, T]$ except at $\left.t_{0} \in\right] 0, T[$ with $\xi_{t_{0}}<\xi_{t_{0}-}$. Note that the function $\xi$ is not l.u.s.c. at time $t_{0}$. We set $Y_{t}:=\sup _{s \geq t} \xi_{s}$ and $A_{t}:=Y_{0}-Y_{t}$, for all $t \in[0, T]$. By the classical Skorokhod's problem (cf. also [12]), $(Y, 0,0, A)$ is the unique solution to the RBSDE with driver $f \equiv 0$ and obstacle $\xi$. We have $Y_{t}=\xi_{t_{0}-}$, if $t<t_{0}$, and $Y_{t}=\xi_{t}$, if $t \geq t_{0}$. Let $\tau_{0}^{*}:=\inf \left\{u \geq 0: Y_{u}=\xi_{u}\right\}$. We have $\tau_{0}^{*}=t_{0}$. Note that here $\Delta A_{\tau_{0}^{*}}=\Delta A_{t_{0}}=\xi_{t_{0}-}-\xi_{t_{0}}>0$. However, $\tau_{0}^{*}=t_{0}$ is not optimal for $Y_{0}=\sup _{s \geq 0} \xi_{s}=\xi_{t_{0}-}$ because $\xi_{t_{0}}<\xi_{t_{0}-}$. In fact, there does not exist an optimal stopping time for $Y_{0}$.

Let us now consider the case where, instead of being decreasing on $\left[t_{0}, T\right]$, the function $\xi$ is increasing on $\left[t_{0}, T\right]$ with $\xi_{T}=\xi_{t_{0}-}$. Note that, again, the function $\xi$ is not l.u.s.c. For each $t \in[0, T], Y_{t}=\sup _{s>t} \xi_{s}=\xi_{t_{0}-}$. The process $A$ is constant equal to 0 , and $\tau_{0}^{*}=T$ is optimal for $Y_{0}$ (and also for $Y_{t}$, for all $t \in[0, T]$ ).

\section{REFERENCES}

[1] Alario-Nazaret M., J.-P. Lepeltier, and B. Marchal (1982): Dynkin games, in Stochastic Differential Systems (Bad Honnef Workshop on stochastic processes), Lecture Notes in Control and Information Sciences 43, Springer-Verlag, Berlin, 23-32.

[2] Barrieu P. and N. El Karoui (2004): Optimal derivatives design under dynamic risk measures, Mathematics of Finance, Contemporary Mathematics (A.M.S. Proceedings), 13-26.

[3] Bayraktar E., I. Karatzas, and S. Yao (2010): Optimal Stopping for Dynamic Convex Risk Measures, Illinois Journal of Mathematics , 54 (3), 1025-1067.

[4] Bismut J.-M. (1973): Conjugate convex functions in optimal stochastic control, Journal of Mathematical Analysis and Applications 44(2), 384-404.

[5] Bouchard B., D. Possamaï, and X. Tan (2016): A general Doob-Meyer-Mertens decomposition for $g$-supermartingale system, Electronic Journal of Probability 21, paper no. 36, 21 pages.

[6] Crépey S. and A. Matoussi (2008): Reflected and doubly reflected BSDEs with jumps: a priori estimates and comparison, Annals of Applied Probability, 18(5), 2041-2069. 
[7] Dellacherie C. and E. Lenglart (1981): Sur des problèmes de régularisation, de recollement et d'interpolation en théorie des processus, Sém. de Proba. XVI, lect. notes in Mathematics, 920, 298-313, Springer-Verlag.

[8] Dellacherie C. and P.-A. Meyer (1975): Probabilité et Potentiel, Chap. I-IV. Nouvelle édition. Hermann.

[9] Dellacherie C. and P.-A. Meyer (1980): Probabilités et Potentiel, Théorie des Martingales, Chap. VVIII. Nouvelle édition. Hermann.

[10] Dumitrescu R., M.-C. Quenez, and A. Sulem (2016): Generalized Dynkin Games and Doubly reflected BSDEs with jumps, to appear in Electronic Journal of Probability.

[11] El Karoui N. (1981): Les aspects probabilistes du contrôle stochastique. École d'été de Probabilités de Saint-Flour IX-1979 Lect. Notes in Math. 876, 73-238. MR0637469

[12] El Karoui N., Kapoudjian C., Pardoux E., Peng S. and M.-C. Quenez (1997): Reflected solutions of Backward SDE's and related obstacle problems for PDE's, The Annals of Probability, 25(2), 702-737.

[13] El Karoui N., Peng S. and M.C. Quenez (1997): Backward Stochastic Differential Equations in Finance, Mathematical Finance 7(1), 1-71.

[14] El Karoui N. and M.-C. Quenez (1997): Non-linear Pricing Theory and Backward Stochastic Differential Equations, Lect. Notes in Mathematics 1656, Ed. W. Runggaldier, Springer.

[15] Essaky H. (2008): Reflected backward stochastic differential equation with jumps and RCLL obstacle. Bulletin des Sciences Mathématiques 132, 690-710.

[16] Gal'chouk L. I. (1981) : Optional martingales, Math. USSR Sbornik 40(4), 435-468.

[17] Grigorova M., P. Imkeller, Y. Ouknine, and M.-C. Quenez (2016): Doubly Reflected BSDEs and Dynkin games: beyond the right-continuous case, working paper.

[18] Hamadène S. (2002) Reflected BSDE's with discontinuous barrier and application, Stochastics and Stochastic Reports 74(3-4), 571-596.

[19] Hamadène S. and Y. Ouknine (2003): Backward stochastic differential equations with jumps and random obstacle, Electronic Journal of Probability 8, 1-20.

[20] Hamadène S. and Y. Ouknine (2015): Reflected backward SDEs with general jumps, Teor. Veroyatnost. i Primenen. , 60(2), 357-376.

[21] Karatzas I. and S. E. Shreve, Methods of mathematical finance, Applications of Mathematics (New York), 39, Springer, New York, 1998.

[22] Kobylanski M. and M.-C. Quenez (2012): Optimal stopping time problem in a general framework, Electronic Journal of Probability 17, 1-28.

[23] Kobylanski M. and M.-C. Quenez (2016): Erratum: Optimal stopping time problem in a general framework, available at https://hal.archives-ouvertes.fr/hal-01328196.

[24] Kobylanski M., M.-C. Quenez, and M. Roger de Campagnolle (2014): Dynkin games in a general framework, Stochastics 86(2), 304-329.

[25] Kobylanski M., M.-C. Quenez and E. Rouy-Mironescu, Optimal multiple stopping time problem, Ann. Appl. Probab. 21 (2011), no. 4, 1365-1399. MR2857451 (2012h:60130)

[26] Lenglart E. (1980): Tribus de Meyer et théorie des processus, Séminaire de probabilités de Strasbourg XIV 1978/79, Lecture Notes in Mathematics Vol. 784, 500-546.

[27] Maingueneau M. A. (1977): Temps d'arrêt optimaux et théorie générale, Séminaire de Probabilités, XII de Strasbourg, 1976/77, 457-467, Lecture Notes in Math., 649 Springer, Berlin.

[28] Ouknine Y. (1998) : Reflected backward stochastic differential equation with jumps, Stochastics and Stoch. Reports 65, 111-125. 
[29] Pardoux E. and S. Peng (1990): Adapted solution of backward stochastic differential equation, Systems E) Control Letters 14, 55Ü61.

[30] Pardoux E. and S. Peng (1992): Backward Stochastic Differential equations and Quasilinear Parabolic Partial Differential equations, Lect. Notes in CIS 176, 200-217.

[31] Peng S. (1999): Monotonic limit theorem of BSDE and nonlinear decomposition theorem of DoobMeyer's type, Probability Theory and Related Fields, 113(4), 473-499.

[32] Peng S. (2004): Nonlinear expectations, nonlinear evaluations and risk measures, 165-253, Lecture Notes in Math., 1856, Springer, Berlin.

[33] Quenez M-C. and A. Sulem (2013): BSDEs with jumps, optimization and applications to dynamic risk measures. Stochastic Processes and Their Applications 123, 0-29.

[34] Quenez M.-C. and A. Sulem (2014): Reflected BSDEs and robust optimal stopping for dynamic risk measures with jumps, Stochastic Processes and their Applications 124(9), 3031-3054.

[35] Rosazza-Gianin E. (2006): Risk measures via g-expectations, Insurance: Mathematics and Economics 39(1), 19-34.

[36] Royer M. (2006): Backward stochastic differential equations with jumps and related non-linear expectations, Stochastic Processes and Their Applications 116, 1358-1376.

[37] Tang S.H. and X. Li (1994): Necessary conditions for optimal control of stochastic systems with random jumps, SIAM J. Cont. and Optim. 32, 1447-1475.

Institut FÜr MATHEMATiK

Humboldt Universität Zu Berlin

UNTER DEN LINDEN 6

10099 Berlin, Germany

E-MAIL: miryana.grigorova@hu-berlin.de imkeller@math.hu-berlin.de

Département de Mathématiques

Faculté des Sciences Semlalia

Université Cadi Ayyad

B.P. 2390

Marrakech, Morocco

E-MAIL: ouknine@ucam.ac.ma
University of Botswana

Private Bag UB 00704

Gaborone, Botswana

E-MAIL: offen@mopipi.ub.bw

Laboratoire de Probabilités

et Modèles Aléatoires

Université Paris-Diderot

BoÎte COURrier 7012

75251 Paris Cedex 05, France

E-MAIL: quenez@math.univ-paris-diderot.fr 